\title{
CERTAIN RESIDUAL PROPERTIES OF HNN-EXTENSIONS WITH CENTRAL ASSOCIATED SUBGROUPS
}

\author{
E. V. SOKOLOV
}

\begin{abstract}
Suppose that $G$ is a group, $H$ and $K$ are proper isomorphic central subgroups of $G$, and $\mathfrak{G}$ is an HNN-extension of $G$ with the associated subgroups $H$ and $K$. We prove necessary and sufficient conditions for $\mathfrak{G}$ to be residually a $\mathcal{C}$-group, where $\mathcal{C}$ is a class of groups closed under taking subgroups, extensions, homomorphic images, and Cartesian products of the form $\prod_{y \in Y} X_{y}$, where $X, Y \in \mathcal{C}$ and $X_{y}$ is an isomorphic copy of $X$ for each $y \in Y$.
\end{abstract}

\section{Introduction. Statement of Results}

Let $\mathcal{C}$ be a class of groups. Recall that a group $X$ is said to be residually a $\mathcal{C}$-group if, for any non-trivial element $x \in X$, there is a homomorphism $\sigma$ of $X$ onto a group from $\mathcal{C}$ such that $x \sigma \neq 1$.

The main question in the study of the $\mathcal{C}$-residuality of HNN-extensions is whether an $\mathrm{HNN}$-extension of a residually $\mathcal{C}$-group is again residually a $\mathcal{C}$-group. All known answers to this question are obtained under various restrictions imposed on the base group, the associated subgroups, and / or the isomorphism between them (the terminology for HNN-extensions used here and below follows [9]). One such restriction is the centrality of the associated subgroups in the base group; the $\mathcal{C}$-residuality of HNN-extensions of this type (including the case of an abelian base group) is studied in [2,6,8,11-15,23,24,33,34].

In $[2,14,15]$, S. Andreadakis, E. Raptis, and D. Varsos give criteria for the residual finiteness and the residual nilpotence of an HNN-extension of a finitely generated abelian group and prove that such an extension is residually solvable. D. I. Moldavanskii $[11,12]$ significantly strengthens some of these results by generalizing them to the case of HNN-extension with central associated subgroups. He proposes an original approach to the study of the $\mathcal{C}$-residuality of such $\mathrm{HNN}$-extensions, which he call the method of descent and ascent of compatible subgroups. In $[11,12]$, this method is applied under the assumption that $\mathcal{C}$ is the class of all finite groups or all finite $p$-groups, where $p$ is a prime number. The aim of this paper is to generalize the results obtained in $[11,12]$ to the case when $\mathcal{C}$ is an arbitrary root class of groups closed under taking quotient groups.

The notion of a root class was introduced by K. Gruenberg [7], and its equivalent definitions are given in [17]. In accordance with one of them, a class of groups $\mathcal{C}$ containing at least one non-trivial group is called root if it is closed under taking subgroups, extensions, and Cartesian products of the form $\prod_{y \in Y} X_{y}$, where $X, Y \in \mathcal{C}$ and $X_{y}$ is an isomorphic copy of $X$ for each $y \in Y$. The examples of root classes are the above-mentioned classes of all finite groups and all finite $p$-groups, as well as the classes of periodic $\pi$-groups of finite exponent (where $\pi$ is a non-empty set of primes), all solvable groups, and all torsion-free groups. We note also that the intersection of any number of root classes is again a root class [17].

The notion of a root class turns out to be very useful in studying the residual properties of free constructions of groups allowing one to prove many statements at once using the same argument. The papers [3,7] are the starting point for these studies, the latest

Key words and phrases. Residual finiteness, residual p-finiteness, residual solvability, root-class residuality, HNN-extension. 
results obtained in this area can be found in [19-21,24-27,31,32]. The root-class residuality of HNN-extensions is studied in [6, 23, 24, 28-30,32]. These papers deal mainly with the cases when the associated subgroups coincide or intersect trivially.

Let us call a sequence $(X, Y, Z, \psi)$ an $H N N$-tuple if $X$ is a group, $Y$ and $Z$ are isomorphic subgroups of $X$, and $\psi: Y \rightarrow Z$ is an isomorphism. If $(X, Y, Z, \psi)$ is an HNN-tuple, then by $\operatorname{HNN}(X, Y, Z, \psi)$ we denote the HNN-extension $\left\langle X, t ; t^{-1} Y t=Z, \psi\right\rangle$. Recall that $\operatorname{HNN}(X, Y, Z, \psi)$ is the group whose generators are the generators of $X$ and the symbol $t$, and whose defining relations are the relations of $X$ and all possible relations of the form $t^{-1} y t=y \psi$, where $y$ and $y \psi$ are words in the generators of $X$ defining an element $y \in Y$ and its image under $\psi$.

Throughout this section, we assume that $(G, H, K, \varphi)$ is an HNN-tuple, $H$ and $K$

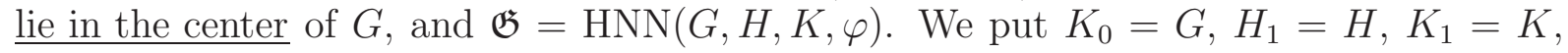
and (if $H_{i}$ and $K_{i}$ are already defined) $H_{i+1}=H_{i} \cap K_{i}, K_{i+1}=H_{i+1} \varphi$. To simplify the notation, the restriction of $\varphi$ to $H_{i}(i \geqslant 1)$ or some other subgroup is denoted below by the same symbol $\varphi$.

Since, for any $i \geqslant 0$, the sequence $\left(K_{i}, H_{i+1}, K_{i+1}, \varphi\right)$ is an HNN-tuple, the group $\mathfrak{K}_{i}=\operatorname{HNN}\left(K_{i}, H_{i+1}, K_{i+1}, \varphi\right)$ is defined. Obviously, if $H_{n}=K_{n}$ for some $n \geqslant 1$, then $H_{n+1}=K_{n}=K_{n+1}$, and therefore $\mathfrak{K}_{n}$ is a split extension of $K_{n}$ by the infinite cyclic group $\langle t\rangle$. It is also easy to see that the restriction of $\varphi$ to $H_{n}$ turns out to be an automorphism of this subgroup, and $\mathfrak{K}_{n}$ is isomorphic to the subgroup $E=\operatorname{sgp}\left\{H_{n}, t\right\}$ of $G$, which is a split extension of $H_{n}$ by $\langle t\rangle$.

The method of descent and ascent of compatible subgroups essentially consists in proving that, under certain conditions, for each $i \geqslant 0$, the $\mathcal{C}$-residuality of $G$ is equivalent to the $\mathcal{C}$-residuality of $\mathfrak{K}_{i}$. If $H_{n}=K_{n}$ for some $n \geqslant 1$, this allows us to reduce the question of the $\mathcal{C}$-residuality of $G$ to the much simpler problem of finding conditions for the split extension $\mathfrak{K}_{n}$ to be residually a $\mathcal{C}$-group. Two criteria for the root-class residuality of split extensions are given at the end of Section 5 .

We note that the equality $H_{n}=K_{n}$ may not hold for any $n$. To guarantee its fulfillment, the theorems given below impose a weaker condition on $G$ : $H_{n}=H_{n+1}$ for some $n$. Corollaries 1-4 describe a number of situations in which the last relation certainly takes place.

Throughout the paper, if $\mathcal{C}$ is a class of groups and $X$ is a group, then $\mathcal{C}^{*}(X)$ denotes the family of normal subgroups of $X$ such that $Y \in \mathcal{C}^{*}(X)$ whenever $X / Y \in \mathcal{C}$. If $\mathcal{C}$ consists only of periodic groups, then we denote by $\pi(\mathcal{C})$ the set of all the prime numbers that divide the orders of the elements of all possible $\mathcal{C}$-groups. Recall that if $\pi$ is a set of primes, then a $\pi$-number is an integer all of whose prime divisors belong to $\pi$, and a $\pi$-group is a periodic group in which the order of each element is a $\pi$-number.

Theorem 1. Suppose that $\mathcal{C}$ is a root class of groups closed under taking quotient groups, $G$ is residually a $\mathcal{C}$-group, and there exists a subgroup $Q \in \mathcal{C}^{*}(G)$ satisfying at least one of the following conditions:

( $\alpha) H \cap Q=1=K \cap Q$,

( $\beta) Q \leqslant H \cap K$ and $Q \varphi=Q$.

I. If $\mathcal{C}$ contains non-periodic groups, then $\mathfrak{G}$ is residually a $\mathcal{C}$-group.

II. If $\mathcal{C}$ consists only of periodic groups, $H \neq G \neq K$, and $H_{n}=H_{n+1}$ for some $n \geqslant 1$, then $\mathfrak{G}$ is residually a $\mathcal{C}$-group if and only if

1) $H_{n}=K_{n}$;

2) the subgroup $E=\operatorname{sgp}\left\{H_{n}, t\right\}$ is residually a $\mathcal{C}$-group.

Corollary 1. If $\mathcal{C}$ is a root class of groups closed under taking quotient groups, $G$ is residually a $\mathcal{C}$-group, $H$ and $K$ are finite, then $H_{n}=K_{n}$ for some $n \geqslant 1$ and the following statements hold. 
I. If $\mathcal{C}$ contains non-periodic groups, then $\mathfrak{G}$ is residually a $\mathcal{C}$-group.

II. If $\mathcal{C}$ consists only of periodic groups, then $\mathfrak{G}$ is residually a $\mathcal{C}$-group if and only if the order of the automorphism $\varphi$ of $H_{n}$ is a $\pi(\mathcal{C})$-number.

Corollary 2. Suppose that $\mathcal{C}$ is a root class of groups closed under taking quotient groups, $H$ and $K$ have finite index in $G$, and $H \neq G \neq K$.

I. If $\mathcal{C}$ contains non-periodic groups, then $\mathfrak{G}$ is residually a $\mathcal{C}$-group if and only if $G$ is residually a $\mathcal{C}$-group and there exists a subgroup $Q \in \mathcal{C}^{*}(G)$ satisfying the conditions $Q \leqslant H \cap K$ and $Q \varphi=Q$.

II. If $\mathcal{C}$ consists only of finite groups, then $\mathfrak{G}$ is residually a $\mathcal{C}$-group if and only if

1) $G / H \in \mathcal{C}$ and $G / K \in \mathcal{C}$;

2) $H_{n}=K_{n}$ for some $n \geqslant 1$;

3) the subgroup $E=\operatorname{sgp}\left\{H_{n}, t\right\}$ is residually a $\mathcal{C}$-group.

Let $\pi$ be a set of primes. Following [16], we call an abelian group $A \pi$-bounded if, for any quotient group $B$ of $A$ and for any $p \in \pi$, the $p$-primary component of $B$ is finite. A nilpotent (solvable) group is said to be $\pi$-bounded if it has a finite central (respectively, subnormal) series with abelian $\pi$-bounded factors. It is easy to see that, for any set $\pi$ of prime numbers, a finitely generated nilpotent group is $\pi$-bounded nilpotent and a polycyclic group is $\pi$-bounded solvable. It is also known (see Proposition 6.7 below) that if a $\pi$-bounded solvable group is abelian, then it belongs to the class of $\pi$-bounded abelian groups. Therefore, we can say that an abelian group is $\pi$-bounded without specifying the class of $\pi$-bounded groups (abelian, nilpotent, or solvable) that we mean.

Throughout the paper, we denote by $\pi^{\prime}$ the set of all primes that do not belong to the set $\pi$. Recall that a subgroup $Y$ of a group $X$ is said to be $\pi^{\prime}$-isolated in this group if, for any $x \in X$ and for any $q \in \pi^{\prime}$, it follows from the inclusion $x^{q} \in Y$ that $x \in Y$. Obviously, if $\pi$ contains all primes, then any subgroup turns out to be $\pi^{\prime}$-isolated.

It is easy to see that the intersection of any number of $\pi^{\prime}$-isolated subgroups is again a $\pi^{\prime}$-isolated subgroup. Therefore, for any subgroup $Y \leqslant X$, there exists the smallest $\pi^{\prime}$-isolated subgroup containing it. We call this subgroup the $\pi^{\prime}$-isolator of $Y$ in $X$ and denote it by $\mathcal{I}_{\pi^{\prime}}(X, Y)$.

Let $\mathcal{C}$ be a class of groups. Recall that a subgroup $Y$ is said to be $\mathcal{C}$-separable in a group $X$ if, for any $x \in X \backslash Y$, there exists a homomorphism $\sigma$ of $X$ onto a group from $\mathcal{C}$ such that $x \sigma \notin Y \sigma[10]$. Obviously, the $\mathcal{C}$-residuality of $X$ is equivalent to the $\mathcal{C}$-separability of its trivial subgroup. It is also known (see Proposition 6.3 below) that if $\mathcal{C}$ consists only of periodic groups, then any $\mathcal{C}$-separable subgroup of $X$ is $\pi(\mathcal{C})^{\prime}$-isolated in this group. Thus, for such a class of groups $\mathcal{C}$, the main problem in the study of $\mathcal{C}$-separability is the search for conditions under which a $\pi(\mathcal{C})^{\prime}$-isolated subgroup turns out to be $\mathcal{C}$-separable.

Theorem 2. Suppose that $\mathcal{C}$ is a root class of groups consisting only of periodic groups and closed under taking quotient groups, $G$ is residually a $\mathcal{C}$-group, $H$ and $K$ are $\pi(\mathcal{C})$-bounded, and $H \neq G \neq K$. Suppose also that $H_{n}=H_{n+1}$ for some $n \geqslant 1$ and, for any $i \in\{0,1, \ldots, n-1\}$ and $N \in \mathcal{C}^{*}\left(H_{i+1} K_{i+1}\right)$, the subgroup $\mathcal{I}_{\pi(\mathcal{C})^{\prime}}\left(K_{i}, N\right)$ is $\mathcal{C}$-separable in $K_{i}$. Then $\mathfrak{G}$ is residually a $\mathcal{C}$-group if and only if

1) $H_{n}=K_{n}$

2) the subgroup $E=\operatorname{sgp}\left\{H_{n}, t\right\}$ is residually a $\mathcal{C}$-group;

3) $H$ and $K$ are $\pi(\mathcal{C})^{\prime}$-isolated in $G$.

Corollary 3. Suppose that $\mathcal{C}$ is a root class of groups consisting only of periodic groups and closed under taking quotient groups, $G$ is $\pi(\mathcal{C})$-bounded nilpotent, $H \neq G \neq K$. Suppose also that there exists a number $m \geqslant 0$ such that $H_{m+1}$ and $K_{m+1}$ are finitely 
generated or $\pi^{\prime}$-isolated in $K_{m}$ for some finite subset $\pi$ of $\pi(\mathcal{C})$. Then $\mathfrak{G}$ is residually $a \mathcal{C}$-group if and only if

1) $H_{n}=K_{n}$ for some $n \geqslant 1$;

2) the subgroup $E=\operatorname{sgp}\left\{H_{n}, t\right\}$ is residually a $\mathcal{C}$-group;

3) $G$ has no $\pi(\mathcal{C})^{\prime}$-torsion, $H$ and $K$ are $\pi(\mathcal{C})^{\prime}$-isolated in $G$.

Corollary 4. Suppose that $\mathcal{C}$ is a root class of groups consisting only of periodic groups and closed under taking quotient groups, $\pi(\mathcal{C})$ contains all primes, $G$ is $\pi(\mathcal{C})$-bounded solvable, and $H \neq G \neq K$. Suppose also that there exists a number $m \geqslant 0$ such that $H_{m+1}$ and $K_{m+1}$ are finitely generated or $\pi^{\prime}$-isolated in $K_{m}$ for some finite set of primes $\pi$. Then $\mathfrak{G}$ is residually a $\mathcal{C}$-group if and only if $H_{n}=K_{n}$ for some $n \geqslant 1$.

Theorems 1 and 2 formulated above are in fact corollaries of Theorems 3 and 4, which are given below and use the notion of regularity of a group with respect to a subgroup.

Suppose that $\mathcal{C}$ is a class of groups, $X$ is a group, and $Y$ is a subgroup of $X$. We say that $X$ is $\mathcal{C}$-regular with respect to $Y$ if, for any subgroup $M \in \mathcal{C}^{*}(Y)$, there exists a subgroup $N \in \mathcal{C}^{*}(X)$ such that $M=N \cap Y$. The notion of regularity generalizes the classical notion of a potent element [1]: if $\mathcal{F}$ is the class of all finite groups, then an element $x \in X$ is potent if and only if $X$ is $\mathcal{F}$-regular with respect to the cyclic subgroup $\langle x\rangle$.

Theorem 3. Suppose that $\mathcal{C}$ is a root class of groups consisting only of periodic groups and closed under taking quotient groups, $G$ is residually a $\mathcal{C}$-group, $H \neq G \neq K$, and $H_{n}=H_{n+1}$ for some $n \geqslant 1$. Suppose also that, for any $i \in\{0,1, \ldots, n-1\}$, $K_{i}$ is $\mathcal{C}$-regular with respect to $H_{i+1} K_{i+1}$ and $\mathcal{I}_{\pi(\mathcal{C})^{\prime}}\left(K_{i}, H_{i+1} K_{i+1}\right)$ is $\mathcal{C}$-separable in $K_{i}$. Then $\mathfrak{G}$ is residually a $\mathcal{C}$-group if and only if

1) $H_{n}=K_{n}$

2) the subgroup $E=\operatorname{sgp}\left\{H_{n}, t\right\}$ is residually a $\mathcal{C}$-group;

3) $H$ and $K$ are $\pi(\mathcal{C})^{\prime}$-isolated in $G$.

Theorem 4. Suppose that $\mathcal{C}$ is a root class of groups containing non-periodic groups and closed under taking quotient groups, $G$ is residually a $\mathcal{C}$-group, and, for some $n \geqslant 0$, there exists a subgroup $Q \in \mathcal{C}^{*}\left(K_{n}\right)$ satisfying at least one of the following conditions:

( $\alpha) H_{n+1} \cap Q=1=K_{n+1} \cap Q$,

( $\beta) Q \leqslant H_{n+1} \cap K_{n+1}$ and $Q \varphi=Q$.

Suppose also that, for any $i \in\{0,1, \ldots, n-1\}, K_{i}$ is $\mathcal{C}$-regular with respect to $H_{i+1} K_{i+1}$ and $H_{i+1} K_{i+1}$ is $\mathcal{C}$-separable in $K_{i}$. Then $\mathfrak{G}$ is residually a $\mathcal{C}$-group.

The above theorems and corollaries generalize the main results from $[2,6,23]$ (as for [2], in the part concerning non-ascending HNN-extensions), as well as Theorem 1.1 from [14] and Theorem 3 from [24]. However, the results mentioned are easier to formulate due to the additional restrictions imposed on the base group and the associated subgroups. The proofs of Theorems 1-4 and Corollaries 1-4 are given in Sections 2-6.

\section{Generalized direct products of Groups}

Let $\Gamma=(V, E)$ be a non-empty connected undirected graph with a vertex set $V$ and an edge set $E$. It is assumed that $\Gamma$ is not necessarily finite, but has no multiple edges and loops. Let us assign to each vertex $v \in V$ a group $G_{v}$ and to each edge $e=\{v, w\} \in E$ a group $H_{e}$ and injective homomorphisms $\varphi_{e, v}: H_{e} \rightarrow G_{v}, \varphi_{e, w}: H_{e} \rightarrow G_{w}$. As a result, we get a graph of groups $\mathcal{G}(\Gamma)$. We call the groups $G_{v}(v \in V), H_{e}(e \in E)$, the subgroups $H_{e} \varphi_{e, v}$, and the homomorphisms $\varphi_{e, v}(e \in E, v \in e)$ the vertex and edge groups, the edge subgroups, and the edge homomorphisms, respectively. 
Consider the group

$$
\begin{aligned}
\operatorname{GDP}(\mathcal{G}(\Gamma))=\left\langle G_{\lambda}(\lambda \in V) ;\left[G_{\mu}, G_{\eta}\right]=1(\mu, \eta \in V, \mu \neq \eta),\right. \\
\left.H_{e} \varphi_{e, v}=H_{e} \varphi_{e, w}(e=\{v, w\} \in E)\right\rangle,
\end{aligned}
$$

whose generators are the generators of $G_{\lambda}(\lambda \in V)$, and whose defining relations are the relations of $G_{\lambda}(\lambda \in V)$ and all possible relations of the form

$$
\left[g_{\mu}, g_{\eta}\right]=1(\mu, \eta \in V, \mu \neq \eta), \quad h_{e} \varphi_{e, v}=h_{e} \varphi_{e, w}\left(e=\{v, w\} \in E, h_{e} \in H_{e}\right),
$$

where $g_{\mu}$ and $g_{\eta}$ are arbitrary words in the generators of $G_{\mu}$ and $G_{\eta}$, respectively, $h_{e} \varphi_{e, v}$ and $h_{e} \varphi_{e, w}$ are some words in the generators of $G_{v}$ and $G_{w}$ that define (in these groups) the images of $h_{e}$ under $\varphi_{e, v}$ and $\varphi_{e, w}$. Following [24], we call the group $\operatorname{GDP}(\mathcal{G}(\Gamma))$ the generalized direct product associated with the graph of groups $\mathcal{G}(\Gamma)$ if

(i) for any $v \in V$, the identity mapping of the generators of $G_{v}$ to $\operatorname{GDP}(\mathcal{G}(\Gamma))$ can be extended to an injective homomorphism, and therefore all the groups $G_{v}(v \in V)$ can be considered subgroups of $\operatorname{GDP}(\mathcal{G}(\Gamma))$;

(ii) for any $e=\{v, w\} \in E$, the equalities $H_{e} \varphi_{e, v}=G_{v} \cap G_{w}=H_{e} \varphi_{e, w}$ hold in $\operatorname{GDP}(\mathcal{G}(\Gamma))$.

We say that the generalized direct product associated with $\mathcal{G}(\Gamma)$ exists if $\operatorname{GDP}(\mathcal{G}(\Gamma))$ satisfies $(i)$ and $(i i)$. Some conditions for the existence of generalized direct products are found in [24]. In particular, the following proposition is proved.

Proposition 2.1. [24, Theorem 1] If $\Gamma$ is a tree and, for any $e \in E$ and $v \in e$, the subgroup $H_{e} \varphi_{e, v}$ lies in the center of $G_{v}$, then the generalized direct product associated with $\mathcal{G}(\Gamma)$ exists.

The main aim of this section is to find conditions for the existence of certain generalized direct products associated with simple cycles.

Let $(X, Y, Z, \psi)$ be an HNN-tuple, and let $\Gamma$ be the simple cycle of length $n \geqslant 3$ with the vertex set $V=\mathbb{Z}_{n}$ and the edge set $E=\left\{\{i-1, i\} \mid i \in \mathbb{Z}_{n}\right\}$. Suppose also that, for any $i \in \mathbb{Z}_{n}, X_{i}$ is an isomorphic copy of $X$ and $\sigma_{i}: X \rightarrow X_{i}$ is an isomorphism. We associate a vertex $i \in \mathbb{Z}_{n}$ with the group $X_{i}$, an edge $e=\{i-1, i\} \in E$ with the group $Y$ and the homomorphisms $\varphi_{e, i-1}=\left.\sigma_{i-1}\right|_{Y}, \varphi_{e, i}=\left.\psi \sigma_{i}\right|_{Z}$ (here and below, all indices are considered modulo $n$ ), and denote the resulting graph of groups by $\mathcal{G}_{n}(X, Y, Z, \psi)$.

Let us call a number $n \geqslant 3$ admissible for an $H N N$-tuple $(X, Y, Z, \psi)$ with a reserve $r$ $(0 \leqslant r \leqslant n-2)$ if the following conditions hold:

$(i)^{\prime}$ for any $i \in \mathbb{Z}_{n}$, the identity mapping of the generators of $X_{i}$ to $\operatorname{GDP}\left(\mathcal{G}_{n}(X, Y, Z, \psi)\right)$ can be extended to an injective homomorphism;

$(\text { ii })^{\prime}$ for any $s \in\{0,1, \ldots, r\}, q \in \mathbb{Z}_{n}, x_{q} \in X_{q}, x_{q+1} \in X_{q+1}, \ldots, x_{q+s+1} \in X_{q+s+1}$, it follows from the equality $x_{q} x_{q+1} \ldots x_{q+s+1}=1$ that $x_{q} \in Y \sigma_{q}$ and $x_{q+s+1} \in Z \sigma_{q+s+1}$.

We note that if a number $n$ is admissible for a tuple $(X, Y, Z, \psi)$ with a reserve $r \in\{0, \ldots, n-2\}$, then the generalized direct product associated with $\mathcal{G}_{n}(X, Y, Z, \psi)$ exists. Indeed, for any $e=\{i-1, i\} \in E$, if $x \in X_{i-1} \cap X_{i}, x_{i-1}=x$, and $x_{i}=x^{-1}$, then $x_{i-1} x_{i}=1$ and, by the admissibility,

$$
x_{i-1} \in Y \sigma_{i-1}=Y \varphi_{e, i-1}, \quad x_{i} \in Z \sigma_{i}=Y \varphi_{e, i} .
$$

Thus, $X_{i-1} \cap X_{i} \leqslant Y \varphi_{e, i-1} \cap Y \varphi_{e, i}$ and therefore $Y \varphi_{e, i-1}=X_{i-1} \cap X_{i}=Y \varphi_{e, i}$.

Proposition 2.2. Suppose that $(G, H, K, \varphi)$ is an $H N N$-tuple, $H$ and $K$ lie in the center of $G, L=H \cap K$, and $M=L \varphi$. Then a number $n \geqslant 3$ is admissible for $(G, H, K, \varphi)$ with a reserve $r \leqslant n-3$ if and only if it is admissible for $(K, L, M, \varphi)$ with the reserve $r+1$. 
Proof. For each $i \in \mathbb{Z}_{n}$, let $G_{i}$ denote an isomorphic copy of $G$, and let $\sigma_{i}: G \rightarrow G_{i}$ be an isomorphism. We put

$$
H_{i}=H \sigma_{i}, \quad K_{i}=K \sigma_{i}, \quad L_{i}=L \sigma_{i}, \quad M_{i}=M \sigma_{i}, \quad \varphi_{i}=\left.\left(\left.\sigma_{i-1}\right|_{H_{i-1}}\right)^{-1} \varphi \sigma_{i}\right|_{K}
$$

(in Section 1, the symbols $H_{i}$ and $K_{i}$ correspond to other subgroups; the notation just introduced is valid only throughout this proof). Then $\varphi_{i}$ is an isomorphism of $H_{i-1}$ onto $K_{i}$, and its restriction to $L_{i-1}$ is an isomorphism of $L_{i-1}$ and $M_{i}$. If $\operatorname{GDP}\left(\mathcal{G}_{n}(G, H, K, \varphi)\right)$ satisfies $(i)^{\prime}$, then it can be considered containing $H_{i}$ and $K_{i}\left(i \in \mathbb{Z}_{n}\right)$, and it follows from the relations $h \sigma_{i-1}=h \varphi \sigma_{i}\left(i \in \mathbb{Z}_{n}, h \in H\right)$ that the equalities $h \varphi_{i}=h\left(i \in \mathbb{Z}_{n}\right.$, $\left.h \in H_{i-1}\right)$ hold in $\operatorname{GDP}\left(\mathcal{G}_{n}(G, H, K, \varphi)\right)$. Similarly, if $\operatorname{GDP}\left(\mathcal{G}_{n}(K, L, M, \varphi)\right)$ satisfies $(i)^{\prime}$, then the equalities $h \varphi_{i}=h\left(i \in \mathbb{Z}_{n}, h \in L_{i-1}\right)$ hold in it.

Sufficiency. We fix a number $i \in \mathbb{Z}_{n}$ and define a mapping $\theta_{i}$ of the subgroup $K_{i} H_{i} \leqslant G_{i}$ to the subgroup $K_{i} K_{i+1} \leqslant \operatorname{GDP}\left(\mathcal{G}_{n}(K, L, M, \varphi)\right)$ as follows: if $h \in H_{i}$ and $k \in K_{i}$, then $(k h) \theta_{i}=k\left(h \varphi_{i+1}\right)$. Let us show that this mapping is well defined and is a subgroup isomorphism extending the identity mapping of $K_{i}$.

If $h_{1}, h_{2} \in H_{i}, k_{1}, k_{2} \in K_{i}$, and $k_{1} h_{1}=k_{2} h_{2}$, then $k_{2}^{-1} k_{1}=h_{2} h_{1}^{-1} \in H_{i} \cap K_{i}=L_{i}$ and therefore the equality $\left(h_{2} h_{1}^{-1}\right) \varphi_{i+1}=h_{2} h_{1}^{-1}$ holds in $\operatorname{GDP}\left(\mathcal{G}_{n}(K, L, M, \varphi)\right)$. Hence,

$$
\begin{aligned}
\left(k_{1} h_{1}\right) \theta_{i}=k_{1}\left(h_{1} \varphi_{i+1}\right)=k_{2}\left(k_{2}^{-1} k_{1}\right) & \left(\left(h_{1} h_{2}^{-1}\right) h_{2}\right) \varphi_{i+1} \\
= & k_{2}\left(k_{2}^{-1} k_{1} h_{1} h_{2}^{-1}\right)\left(h_{2} \varphi_{i+1}\right)=k_{2}\left(h_{2} \varphi_{i+1}\right)=\left(k_{2} h_{2}\right) \theta_{i} .
\end{aligned}
$$

Suppose that $h \in H_{i}, k \in K_{i}$, and $1=(k h) \theta_{i}=k\left(h \varphi_{i+1}\right)$. Since $k \in K_{i}, h \varphi_{i+1} \in K_{i+1}$, and $n$ is admissible for $(K, L, M, \varphi)$, then $k \in L_{i}$ and $h \varphi_{i+1} \in M_{i+1}$. Hence, $h \in L_{i}$ and $1=k\left(h \varphi_{i+1}\right)=k h$ because the equality $h \varphi_{i+1}=h$ holds in $\operatorname{GDP}\left(\mathcal{G}_{n}(K, L, M, \varphi)\right)$.

Thus, $\theta_{i}$ is well defined and injective, it is clear that it is homomorphic and surjective.

Let $\Delta$ be the star graph with the vertex set $\left\{v_{i}\left(i \in \mathbb{Z}_{n}\right), w\right\}$. We associate its central vertex $w$ with the group $\operatorname{GDP}\left(\mathcal{G}_{n}(K, L, M, \varphi)\right)$, the leaf $v_{i}\left(i \in \mathbb{Z}_{n}\right)$ with the group $G_{i}$, the edge $\left\{w, v_{i}\right\}\left(i \in \mathbb{Z}_{n}\right)$ with the subgroup $K_{i} H_{i}$ of $G_{i}$ and the homomorphisms, one of which is the identity mapping and the other coincides with $\theta_{i}$. Let us denote the resulting graph by $\mathcal{G}(\Delta)$.

It follows from the definitions of $\mathcal{G}(\Delta), \theta_{i}\left(i \in \mathbb{Z}_{n}\right)$, and $\varphi_{i}\left(i \in \mathbb{Z}_{n}\right)$ that, for all $i \in \mathbb{Z}_{n}$ and $h \in H$, the equalities $h \sigma_{i-1}=\left(h \sigma_{i-1}\right) \theta_{i-1}=\left(h \sigma_{i-1}\right) \varphi_{i}=h \varphi \sigma_{i}$ hold in $\operatorname{GDP}(\mathcal{G}(\Delta))$. Therefore, the identity mapping of the generators of $\operatorname{GDP}\left(\mathcal{G}_{n}(G, H, K, \varphi)\right)$ to $\operatorname{GDP}(\mathcal{G}(\Delta))$ defines a homomorphism, which we denote by $\lambda$.

Let, for any $i \in \mathbb{Z}_{n}, \alpha_{i}: G_{i} \rightarrow \operatorname{GDP}(\mathcal{G}(\Delta))$ and $\beta_{i}: G_{i} \rightarrow \operatorname{GDP}\left(\mathcal{G}_{n}(G, H, K, \varphi)\right)$ be the homomorphisms defined by the identity mappings of the generators of $G_{i}$. Since the diagram

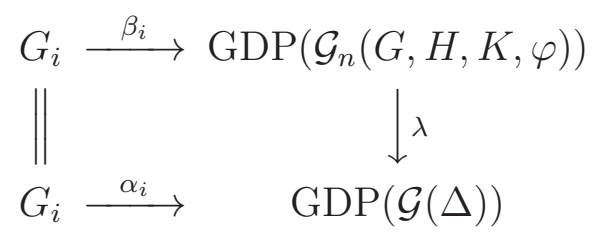

is commutative and $\alpha_{i}$ is injective by Proposition 2.1, then $\beta_{i}$ is also injective and therefore $\operatorname{GDP}\left(\mathcal{G}_{n}(G, H, K, \varphi)\right)$ satisfies $(i)^{\prime}$. Let us verify that this group also satisfies $(i i)^{\prime}$.

Suppose that numbers $q \in \mathbb{Z}_{n}, s \in\{0,1, \ldots, r\}$ and elements

$$
g_{q} \in G_{q}, g_{q+1} \in G_{q+1}, \ldots, g_{q+s+1} \in G_{q+s+1}
$$

are such that $g_{q} g_{q+1} \ldots g_{q+s+1}=1$ in $\operatorname{GDP}\left(\mathcal{G}_{n}(G, H, K, \varphi)\right)$. Then this equality also holds in $\operatorname{GDP}(\mathcal{G}(\Delta))$ by the definition of $\lambda$.

Let $j \in\{0,1, \ldots, s+1\}$, and let $\mathcal{G}_{q+j}$ be the graph of groups obtained from $\mathcal{G}(\Delta)$ by deleting the vertex $v_{q+j}$ and the edge $\left\{v_{q+j}, w\right\}$. Then $\operatorname{GDP}(\mathcal{G}(\Delta))$ is the general- 
ized direct product $\mathfrak{P}_{j}$ of the groups $\operatorname{GDP}\left(\mathcal{G}_{q+j}\right)$ and $G_{q+j}$ with the amalgamated subgroups $K_{q+j} K_{q+j+1}$ and $K_{q+j} H_{q+j}$. It follows from the relation $r \leqslant n-3$ that $s+2<n$ and therefore

$$
\begin{gathered}
g_{q+j-1}^{-1} g_{q+j-2}^{-1} \ldots g_{q}^{-1} g_{q+s+1}^{-1} \ldots g_{q+j+2}^{-1} g_{q+j+1}^{-1} \in \operatorname{GDP}\left(\mathcal{G}_{q+j}\right) \\
g_{q+j}=g_{q+j-1}^{-1} g_{q+j-2}^{-1} \ldots g_{q}^{-1} g_{q+s+1}^{-1} \ldots g_{q+j+2}^{-1} g_{q+j+1}^{-1} \in \operatorname{GDP}\left(\mathcal{G}_{q+j}\right) \cap G_{q+j} .
\end{gathered}
$$

Since $\mathfrak{P}_{j}$ satisfies $\left(\right.$ ii) by Proposition 2.1 , we have $g_{q+j} \in K_{q+j} H_{q+j}$.

For each $j \in\{0,1, \ldots, s+1\}$, let us write the element $g_{q+j}$ in the form $g_{q+j}=k_{q+j} h_{q+j}$, where $h_{q+j} \in H_{q+j}, k_{q+j} \in K_{q+j}$. Since $\operatorname{GDP}\left(\mathcal{G}_{n}(G, H, K, \varphi)\right)$ satisfies $(i)^{\prime}$, then the relations $h_{q+j}=h_{q+j} \varphi_{q+j+1} \in K_{q+j+1}(0 \leqslant j \leqslant s+1)$ hold in it and therefore

$$
k_{q} \in K_{q}, h_{q} k_{q+1} \in K_{q+1}, \ldots, h_{q+s} k_{q+s+1} \in K_{q+s+1}, h_{q+s+1} \in K_{q+s+2} .
$$

As noted above, the equality $g_{q} g_{q+1} \ldots g_{q+s+1}=1$ holds in $\operatorname{GDP}(\mathcal{G}(\Delta))$. We rewrite it in the form

$$
k_{q}\left(h_{q} k_{q+1}\right) \ldots\left(h_{q+s} k_{q+s+1}\right) h_{q+s+1}=1 .
$$

By Proposition 2.1, $\operatorname{GDP}\left(\mathcal{G}_{n}(K, L, M, \varphi)\right)$ is embedded into $\operatorname{GDP}(\mathcal{G}(\Delta))$ by the identity mapping of the generators. Therefore, the last relation holds in $\operatorname{GDP}\left(\mathcal{G}_{n}(K, L, M, \varphi)\right)$, and since $n$ is admissible for $(K, L, M, \varphi)$ with the reserve $r+1$, then $h_{q+s+1} \in M_{q+s+2}$ and $k_{q} \in L_{q}$. Hence, $h_{q+s+1} \varphi_{q+s+2}^{-1} \in L_{q+s+1} \leqslant K_{q+s+1}$ and

$$
g_{q}=k_{q} h_{q} \in H_{q}, \quad g_{q+s+1}=k_{q+s+1} h_{q+s+1}=k_{q+s+1}\left(h_{q+s+1} \varphi_{q+s+2}^{-1}\right) \in K_{q+s+1},
$$

as required.

Necessity. It is easy to see that the mapping of words acting on the generators of the groups $K_{i}\left(i \in \mathbb{Z}_{n}\right)$ as the natural embeddings $\iota_{i}: K_{i} \rightarrow G_{i}\left(i \in \mathbb{Z}_{n}\right)$ takes all the defining relations of $\operatorname{GDP}\left(\mathcal{G}_{n}(K, L, M, \varphi)\right)$ to the equalities valid in $\operatorname{GDP}\left(\mathcal{G}_{n}(G, H, K, \varphi)\right)$ and therefore defines a homomorphism $\mu$ from the first group to the second.

Let $\beta_{i}: G_{i} \rightarrow \operatorname{GDP}\left(\mathcal{G}_{n}(G, H, K, \varphi)\right)$ and $\gamma_{i}: K_{i} \rightarrow \operatorname{GDP}\left(\mathcal{G}_{n}(K, L, M, \varphi)\right)$ be the homomorphisms defined by the identity mappings of the generators of $G_{i}$ and $K_{i}$. Then, for any $i \in \mathbb{Z}_{n}$, the diagram

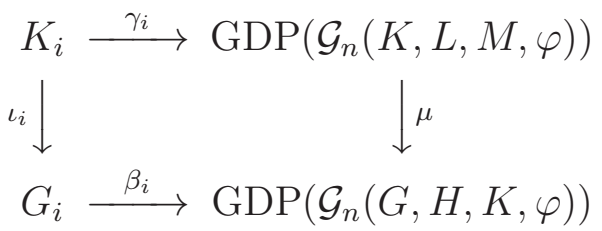

is commutative, and since the homomorphisms $\beta_{i}\left(i \in \mathbb{Z}_{n}\right)$ are injective, the homomorphisms $\gamma_{i}\left(i \in \mathbb{Z}_{n}\right)$ are also injective. Thus, the group $\operatorname{GDP}\left(\mathcal{G}_{n}(K, L, M, \varphi)\right)$ satisfies $(i)^{\prime}$.

Suppose that numbers $q \in \mathbb{Z}_{n}, s \in\{0,1, \ldots, r+1\}$ and elements

$$
k_{q} \in K_{q}, k_{q+1} \in K_{q+1}, \ldots, k_{q+s+1} \in K_{q+s+1}
$$

are such that $k_{q} k_{q+1} \ldots k_{q+s+1}=1$. It follows from the definition of $\mu$ that the last equality also holds in $\operatorname{GDP}\left(\mathcal{G}_{n}(G, H, K, \varphi)\right)$. Since $n$ is admissible for $(G, H, K, \varphi)$ with the reserve $r$ and

$$
k_{q} \in G_{q}, \ldots, k_{q+s-1} \in G_{q+s-1}, k_{q+s} k_{q+s+1}=k_{q+s}\left(k_{q+s+1} \varphi_{q+s+1}^{-1}\right) \in G_{q+s},
$$

then $k_{q} \in H_{q}$ and $k_{q+s}\left(k_{q+s+1} \varphi_{q+s+1}^{-1}\right) \in K_{q+s}$. The first relation means that $k_{q} \in H_{q} \cap K_{q}=L_{q}$, the second one implies that $k_{q+s+1} \varphi_{q+s+1}^{-1} \in K_{q+s}$ and therefore

$$
k_{q+s+1} \varphi_{q+s+1}^{-1} \in H_{q+s} \cap K_{q+s}=L_{q+s} .
$$

Hence, $k_{q+s+1} \in M_{q+s+1}$, as required. 
Proposition 2.3. Suppose that $(G, H, K, \varphi)$ is an HNN-tuple, $G$ is an abelian group, and $H=G=K$. If the order $q$ of the automorphism $\varphi$ is finite and divides a number $n \geqslant 3$, then $n$ is admissible for $(G, H, K, \varphi)$ with any reserve $r \in\{0,1, \ldots, n-2\}$.

Proof. For each $i \in \mathbb{Z}_{n}$, let again $G_{i}$ denote an isomorphic copy of $G$, and let $\sigma_{i}: G \rightarrow G_{i}$ be an isomorphism. Consider the star graph $\Delta$ with the vertex set $\left\{v_{i}\left(i \in \mathbb{Z}_{n}\right), w\right\}$ and associate its central vertex $w$ with the group $G$, the leaf $v_{i}\left(i \in \mathbb{Z}_{n}\right)$ with the group $G_{i}$, and the edge $\left\{w, v_{i}\right\}\left(i \in \mathbb{Z}_{n}\right)$ with the group $G$ and the homomorphisms, one of which is the identity mapping and the other coincides with the isomorphism $\varphi^{i} \sigma_{i}$. Since $q \mid n$, it follows from the relation $x \equiv y(\bmod n)$ that $\varphi^{x}=\varphi^{y}$. Therefore, the notation $\varphi^{i}$ is correct.

We denote the constructed graph of groups by $\mathcal{G}(\Delta)$. It follows from its definition that, for any $i \in \mathbb{Z}_{n}$ and $g^{\prime} \in G$, the equality $g^{\prime}=g^{\prime} \varphi^{i} \sigma_{i}$ holds in $\operatorname{GDP}(\mathcal{G}(\Delta))$ and therefore $g \sigma_{i-1}=g \varphi^{-(i-1)}=g \varphi \sigma_{i}$ for all $i \in \mathbb{Z}_{n}, g \in G$. Hence, the identity mapping of the generators of $\operatorname{GDP}\left(\mathcal{G}_{n}(G, H, K, \varphi)\right)$ to $\operatorname{GDP}(\mathcal{G}(\Delta))$ can be extended to a homomorphism

$$
\lambda: \operatorname{GDP}\left(\mathcal{G}_{n}(G, H, K, \varphi)\right) \rightarrow \operatorname{GDP}(\mathcal{G}(\Delta)) .
$$

Let $\alpha_{i}: G_{i} \rightarrow \operatorname{GDP}(\mathcal{G}(\Delta))$ and $\beta_{i}: G_{i} \rightarrow \operatorname{GDP}\left(\mathcal{G}_{n}(G, H, K, \varphi)\right)$ be the homomorphisms defined by the identity mappings of the generators of $G_{i}\left(i \in \mathbb{Z}_{n}\right)$. Then, for any $i \in \mathbb{Z}_{n}$, the diagram

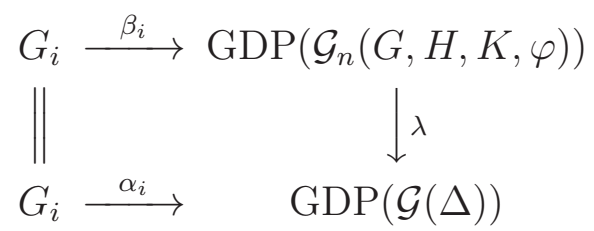

is commutative. Since the homomorphisms $\alpha_{i}\left(i \in \mathbb{Z}_{n}\right)$ are injective by Proposition 2.1, then the homomorphisms $\beta_{i}\left(i \in \mathbb{Z}_{n}\right)$ are also injective. Therefore, $\operatorname{GDP}\left(\mathcal{G}_{n}(G, H, K, \varphi)\right)$ satisfies $(i)^{\prime}$. It follows from the equalities $H=G=K$ that $(i i)^{\prime}$ is satisfied trivially.

Proposition 2.4. Suppose that a number $n \geqslant 3$ is admissible for an $H N N$-tuple $(G, H, K, \varphi)$ with a reserve $r \in\{0,1, \ldots, n-2\}$ and $C_{n}$ is a cyclic group of order $n$ with a generator c. Suppose also that $\mathcal{C}$ is a class of groups closed under taking quotient groups and extensions. If $G \in \mathcal{C}$ and $C_{n} \in \mathcal{C}$, then there exists a homomorphism of the group $\mathfrak{G}=\operatorname{HNN}(G, H, K, \varphi)$ onto a group from $\mathcal{C}$ acting injectively on $G$.

Proof. Let $G_{i}$ and $\sigma_{i}\left(i \in \mathbb{Z}_{n}\right)$ be defined as above. Then the generalized direct product $P=\operatorname{GDP}\left(\mathcal{G}_{n}(G, H, K, \varphi)\right)$ has the representation

$$
P=\left\langle G_{i}\left(i \in \mathbb{Z}_{n}\right) ;\left[G_{i}, G_{j}\right]=1\left(i, j \in \mathbb{Z}_{n}, i \neq j\right), H \sigma_{i-1}=K \sigma_{i}\left(i \in \mathbb{Z}_{n}\right)\right\rangle,
$$

which shows that the map extending the isomorphisms $\sigma_{i}^{-1} \sigma_{i-1}: G_{i} \rightarrow G_{i-1}$ defines an automorphism $\alpha$ of this group. Obviously, the order of this automorphism divides $n$, and therefore there exists a split extension $Q$ of $P$ by $C_{n}$ such that $\left.\hat{c}\right|_{P}=\alpha$ (here and below, $\hat{x}$ denotes the inner automorphism defined by an element $x$ ). Since $G \in \mathcal{C}$, then $P$ is a quotient group of the direct product of $n \mathcal{C}$-groups. Hence, $P \in \mathcal{C}$ and $Q \in \mathcal{C}$ because $\mathcal{C}$ is closed under taking quotient groups and extensions.

It is easy to see that the map $\rho: \mathfrak{G} \rightarrow Q$ extending the homomorphism $\sigma_{0}: G \rightarrow G_{0}$ and mapping $t$ to $c$ takes all the defining relations of $\mathfrak{G}$ to the equalities valid in $Q$ and therefore is a homomorphism. Since $c^{i} G_{0} c^{-i}=G_{i}\left(i \in \mathbb{Z}_{n}\right)$, this homomorphism is surjective. It remains to note that, because $n$ is admissible, $\rho$ is injective on $G$ and thus is the required mapping. 


\section{Compatible subgroups}

Let $(G, H, K, \varphi)$ be an HNN-tuple, and let $\mathfrak{G}=\operatorname{HNN}(G, H, K, \varphi)$. Recall that a subgroup $N \leqslant G$ is said to be $(H, K, \varphi)$-compatible if $(N \cap H) \varphi=N \cap K$.

It is easy to verify that if a subgroup $N$ is normal in $G$ and is $(H, K, \varphi)$-compatible, then the mapping $\varphi_{N}: H N / N \rightarrow K N / N$ taking a coset $h N(h \in H)$ to $(h \varphi) N$ is well defined and is a subgroup isomorphism. Therefore, the sequence

$$
\left(G / N, H N / N, K N / N, \varphi_{N}\right)
$$

turns out to be an HNN-tuple. It is also easy to see that the map

$$
\rho_{N}: \mathfrak{G} \rightarrow \operatorname{HNN}\left(G / N, H N / N, K N / N, \varphi_{N}\right)
$$

extending the natural homomorphism $G \rightarrow G / N$ and taking $t$ to $t$ is a surjective homomorphism, and its kernel coincides with the normal closure of $N$ in $\mathfrak{G}$.

For every class of groups $\mathcal{C}$, consider three families of subgroups. Namely, suppose that

- $\mathcal{C}^{*}(G, H, K, \varphi)$ is the family of all $(H, K, \varphi)$-compatible subgroups from $\mathcal{C}^{*}(G)$;

- $\mathcal{C}_{r}^{*}(G, H, K, \varphi)(r \geqslant 0)$ is the subset of $\mathcal{C}^{*}(G, H, K, \varphi)$ defined as follows: a subgroup $N \in \mathcal{C}^{*}(G, H, K, \varphi)$ belongs to $\mathcal{C}_{r}^{*}(G, H, K, \varphi)$ if and only if there exists a number $n \geqslant \max \{3, r+2\}$ such that $\mathcal{C}$ contains a cyclic group of order $n$ and $n$ is admissible for $\left(G / N, H N / N, K N / N, \varphi_{N}\right)$ with the reserve $r$;

$$
-\mathcal{C}_{\cap}^{*}(G, H, K, \varphi)=\left\{U \cap G \mid U \in \mathcal{C}^{*}(\mathfrak{G})\right\} .
$$

Proposition 3.1. If $\mathcal{C}$ is a class of groups closed under taking subgroups and direct products of a finite number of factors, and $X$ is a group, then the following statements hold.

1. The intersection of a finite number of subgroups from the family $\mathcal{C}^{*}(X)$ again belongs to this family [25, Proposition 2];

2. If $X$ is residually a $\mathcal{C}$-group and $S$ is a finite subset of non-trivial elements of $X$, then there exists a subgroup $Y \in \mathcal{C}^{*}(X)$ such that $Y \cap S=\varnothing$ [32, Proposition 2].

Proposition 3.2. If $\mathcal{C}$ is a class of groups closed under taking subgroups and direct products of a finite number of factors, and $(G, H, K, \varphi)$ is an HNN-tuple, then the families $\mathcal{C}^{*}(G, H, K, \varphi)$ and $\mathcal{C}_{\cap}^{*}(G, H, K, \varphi)$ are closed under taking the intersections of a finite number of subgroups.

Proof. An obvious induction allows us to consider the intersection of only two subgroups. If $N_{1}, N_{2} \in \mathcal{C}^{*}(G, H, K, \varphi)$ and $N=N_{1} \cap N_{2}$, then $N \in \mathcal{C}^{*}(G)$ by Proposition 3.1 and

$$
\begin{aligned}
(N \cap H) \varphi=\left(\left(N_{1} \cap H\right) \cap\left(N_{2} \cap H\right)\right) \varphi=\left(N_{1} \cap H\right) \varphi & \cap\left(N_{2} \cap H\right) \varphi \\
& =\left(N_{1} \cap K\right) \cap\left(N_{2} \cap K\right)=N \cap K
\end{aligned}
$$

because $\varphi$ is injective. Therefore, $N \in \mathcal{C}^{*}(G, H, K, \varphi)$.

Suppose that $N_{1}, N_{2} \in \mathcal{C}_{\cap}^{*}(G, H, K, \varphi), \mathfrak{G}=\operatorname{HNN}(G, H, K, \varphi)$, and $U_{1}, U_{2} \in \mathcal{C}^{*}(\mathfrak{G})$ are subgroups such that $N_{1}=U_{1} \cap G, N_{2}=U_{2} \cap G$. If $N=N_{1} \cap N_{2}$ and $U=U_{1} \cap U_{2}$, then $N=U \cap G$ and, again by Proposition 3.1, $U \in \mathcal{C}^{*}(\mathfrak{G})$. Thus, $N \in \mathcal{C}_{\cap}^{*}(G, H, K, \varphi)$.

Proposition 3.3. [24, Theorem 3] Suppose that $\mathcal{C}$ is a root class of groups containing non-periodic groups and closed under taking quotient groups, $(G, H, K, \varphi)$ is an $H N N$ tuple, and $\mathfrak{G}=\operatorname{HNN}(G, H, K, \varphi)$. Suppose also that $G$ is residually a $\mathcal{C}$-group, $H$ and $K$ lie in the center of $G$, and there exists a homomorphism $\rho$ of $G$ onto a group from $\mathcal{C}$ acting injectively on $H$ and $K$. Then $\rho$ can be extended to a homomorphism of $\mathfrak{G}$ onto a group from $\mathcal{C}$ and $\mathfrak{G}$ is residually a $\mathcal{C}$-group. 
Proposition 3.4. Let $\mathcal{C}$ be a class of groups, and let $(G, H, K, \varphi)$ be an HNN-tuple.

1. If $\mathcal{C}$ is closed under taking subgroups, then $\mathcal{C}_{\cap}^{*}(G, H, K, \varphi) \subseteq \mathcal{C}^{*}(G, H, K, \varphi)$.

2. If $\mathcal{C}$ is a root class of groups containing non-periodic groups and closed under taking quotient groups, $H$ and $K$ lie in the center of $G$, then $\mathcal{C}^{*}(G, H, K, \varphi) \subseteq \mathcal{C}_{\cap}^{*}(G, H, K, \varphi)$.

3. If $\mathcal{C}$ is closed under taking quotient groups and extensions, then $\mathcal{C}_{r}^{*}(G, H, K, \varphi) \subseteq$ $\mathcal{C}_{\cap}^{*}(G, H, K, \varphi)$ for any $r \geqslant 0$.

Proof. 1. Suppose that $\mathfrak{G}=\operatorname{HNN}(G, H, K, \varphi), N \in \mathcal{C}_{\cap}^{*}(G, H, K, \varphi)$, and $U \in \mathcal{C}^{*}(\mathfrak{G})$ is a subgroup such that $N=U \cap G$. Then

$$
G / N=G /(U \cap G) \cong G U / U \leqslant \mathfrak{G} / U
$$

and $G / N \in \mathcal{C}$ because $\mathcal{C}$ is closed under taking subgroups. Since $U$ is normal in $\mathfrak{G}$, then

$$
(U \cap H) \varphi=t^{-1}(U \cap H) t \leqslant t^{-1} U t \cap t^{-1} H t=U \cap H \varphi=U \cap K
$$

and similarly $(U \cap K) \varphi^{-1} \leqslant U \cap H$. Hence,

$$
(N \cap H) \varphi=(U \cap G \cap H) \varphi=(U \cap H) \varphi=U \cap K=U \cap G \cap K=N \cap K
$$

and therefore $N \in \mathcal{C}^{*}(G, H, K, \varphi)$.

2. Let $N \in \mathcal{C}^{*}(G, H, K, \varphi)$. Then the HNN-tuple $\left(G / N, H N / N, K N / N, \varphi_{N}\right)$, the group $\mathfrak{G}_{N}=\operatorname{HNN}\left(G / N, H N / N, K N / N, \varphi_{N}\right)$, and the homomorphism $\rho_{N}: \mathfrak{G} \rightarrow \mathfrak{G}_{N}$ are defined. It follows from the definition of the family $\mathcal{C}^{*}(G, H, K, \varphi)$ that $G / N \in \mathcal{C}$. Hence, by Proposition 3.3, there exists a homomorphism $\sigma$ of $\mathfrak{G}_{N}$ onto a group from $\mathcal{C}$ extending the identity mapping of $G / N$. Let $U_{N}=\operatorname{ker} \sigma$, and let $U$ be the preimage of $U_{N}$ under $\rho_{N}$. Then $U_{N} \in \mathcal{C}^{*}\left(\mathfrak{G}_{N}\right), U_{N} \cap G / N=1$, and $U \in \mathcal{C}^{*}(\mathfrak{G})$. Since $\rho_{N}$ extends the natural homomorphism $G \rightarrow G / N$, then $\operatorname{ker} \rho_{N} \cap G=N$ and $U \cap G \leqslant \operatorname{ker} \rho_{N}$. Therefore, $U \cap G=N$ and $N \in \mathcal{C}_{\cap}^{*}(G, H, K, \varphi)$.

3. If $N \in \mathcal{C}_{r}^{*}(G, H, K, \varphi)$, then, by Proposition 2.4, there exists a homomorphism of the group $\operatorname{HNN}\left(G / N, H N / N, K N / N, \varphi_{N}\right)$ onto a group from $\mathcal{C}$ acting injectively on $G / N$. Hence, we can use exactly the same argument as in the proof of Statement 2.

Proposition 3.5. Let $\mathcal{C}$ be a root class of groups consisting only of periodic groups and closed under taking quotient groups. If $(G, H, K, \varphi)$ is an HNN-tuple, $G$ is an abelian group, $H=G=K$, and $N$ is a subgroup of $G$, then the following statements are equivalent.

1. $N \in \mathcal{C}_{\cap}^{*}(G, H, K, \varphi)$.

2. $N \in \mathcal{C}_{r}^{*}(G, H, K, \varphi)$ for any $r \geqslant 0$.

3. $N \varphi=N, G / N \in \mathcal{C}$, and the order of the automorphism $\varphi_{N}$ of $G / N$ induced by the automorphism $\varphi$ is finite and is a $\pi(\mathcal{C})$-number.

Proof. $1 \Rightarrow 3$. By Proposition 3.4, $N \in \mathcal{C}^{*}(G, H, K, \varphi)$. Hence, $G / N \in \mathcal{C}$, and since $H=G=K$, then

$$
N \varphi=(N \cap H) \varphi=N \cap K=N .
$$

Let $\mathfrak{G}=\operatorname{HNN}(G, H, K, \varphi)$. Because $N \in \mathcal{C}_{\cap}^{*}(G, H, K, \varphi)$, there exists a subgroup $U \in \mathcal{C}^{*}(\mathfrak{G})$ such that $N=U \cap G$. Since $\mathfrak{G} / U \in \mathcal{C}$ and $\mathcal{C}$ consists only of periodic groups, then the order $n$ of $t$ modulo $U$ is finite and is a $\pi(\mathcal{C})$-number.

It follows from the equality $N=U \cap G$ that the mapping $\gamma: G / N \rightarrow \mathfrak{G} / U$ taking a coset $g N$ to $g U$ is well defined and is an injective homomorphism. For any $g \in G$, we have

$$
(g N) \varphi_{N}^{n}=\left(g \varphi^{n}\right) N=(g N) \gamma\left(\widehat{t^{n} U}\right) \gamma^{-1}=g N .
$$

Hence, the order of $\varphi_{N}$ divides $n$ and therefore is a $\pi(\mathcal{C})$-number. 
$3 \Rightarrow 2$. We fix a number $r \geqslant 0$ and choose a $\pi(\mathcal{C})$-number $n>r+3$ to be a multiple of the order of $\varphi_{N}$ (it is possible to find a $\pi(\mathcal{C})$-number greater than $r+3$ because $\mathcal{C}$ contains non-trivial groups and therefore $\pi(\mathcal{C}) \neq \varnothing$ ). Since $H=G=K$, then

$$
(N \cap H) \varphi=N \varphi=N=N \cap K
$$

and hence $N \in \mathcal{C}^{*}(G, H, K, \varphi)$. Therefore, the tuple $\left(G / N, H N / N, K N / N, \varphi_{N}\right)$ is defined and, by Proposition 2.3, $n$ is admissible for this tuple with the reserve $r$. It remains to note that $\mathcal{C}$ contains a cyclic group of order $n$ : this fact follows from the definition of $\pi(\mathcal{C})$ and the assumption that $\mathcal{C}$ is closed under taking subgroups and extensions.

$2 \Rightarrow 1$. This implication is a consequence of Proposition 3.4.

\section{Descent and ascent of compatible subgroups}

The proofs of the propositions in this section follow the ideas of $[11,12]$ and, in some places, repeat the arguments given in the above papers almost word for word.

Proposition 4.1. Suppose that $\mathcal{C}$ is a class of groups closed under taking subgroups, $(G, H, K, \varphi)$ is an HNN-tuple, $L=H \cap K, M=L \varphi$, and $X$ is a subgroup of $G$ containing $L$ and $M$. Suppose also that $N$ is a subgroup of $G$ and $R=N \cap X$. Then the following statements hold.

1. If $N \in \mathcal{C}^{*}(G, H, K, \varphi)$, then $R \in \mathcal{C}^{*}(X, L, M, \varphi)$.

2. If $N \in \mathcal{C}_{\cap}^{*}(G, H, K, \varphi)$, then $R \in \mathcal{C}_{\cap}^{*}(X, L, M, \varphi)$.

Proof. 1. We have $X / R \in \mathcal{C}$ because

$$
X / R=X /(N \cap X) \cong X N / N \leqslant G / N \in \mathcal{C}
$$

and $\mathcal{C}$ is closed under taking subgroups. Since $\varphi$ is injective and $N$ is $(H, K, \varphi)$-compatible, then

$$
((N \cap H) \cap L) \varphi=(N \cap K) \cap M .
$$

It follows from this equality and the relation $L \cup M \leqslant X$ that

$$
\begin{aligned}
(R \cap L) \varphi=((N \cap X) \cap L) \varphi= & (N \cap L) \varphi=((N \cap H) \cap L) \varphi \\
& =(N \cap K) \cap M=N \cap M=(N \cap X) \cap M=R \cap M .
\end{aligned}
$$

Thus, $R \in \mathcal{C}^{*}(X, L, M, \varphi)$.

2. If $\mathfrak{G}=\operatorname{HNN}(G, H, K, \varphi)$ and $\mathfrak{X}=\operatorname{HNN}(X, L, M, \varphi)$, then the map $\lambda: \mathfrak{X} \rightarrow \mathfrak{G}$ taking the generators of $\mathfrak{X}$ to the corresponding elements of $\mathfrak{G}$ defines a homomorphism, which acts identically on $X$. Let $U \in \mathcal{C}^{*}(\mathfrak{G})$ be a subgroup such that $N=U \cap G$, and suppose that $\tilde{\mathfrak{X}}=\mathfrak{X} \lambda, \tilde{V}=U \cap \tilde{\mathfrak{X}}, V$ is the full preimage of $\tilde{V}$ under $\lambda$. Then $\tilde{\mathfrak{X}} / \tilde{V}$ and $\mathfrak{X} / V$ are isomorphic groups, which belong to $\mathcal{C}$ because

$$
\tilde{\mathfrak{X}} / \tilde{V}=\tilde{\mathfrak{X}} /(U \cap \tilde{\mathfrak{X}}) \cong \tilde{\mathfrak{X}} U / U \leqslant \mathfrak{G} / U \in \mathcal{C}
$$

and $\mathcal{C}$ is closed under taking subgroups. Since $\lambda$ acts identically on $X$, it follows from the equalities

$$
\tilde{V} \cap X=U \cap \tilde{\mathfrak{X}} \cap X=U \cap X=U \cap G \cap X=N \cap X=R
$$

that $V \cap X=R$. Therefore, $R \in \mathcal{C}_{\cap}^{*}(X, L, M, \varphi)$.

Let $X$ be a group, and let $Y$ and $Z$ be its subgroups. Recall that a family $\Omega$ of normal subgroups of $X$ is said to be a filtration if $\bigcap_{N \in \Omega} N=1$. A filtration $\Omega$ is called

- a $Y$-filtration if $\bigcap_{N \in \Omega} Y N=1$;

- a $(Y, Z)$-filtration if it is a $Y$-filtration and a $Z$-filtration. 
Proposition 4.2. Suppose that $\mathcal{C}$ is a class of groups closed under taking subgroups, $(G, H, K, \varphi)$ is an HNN-tuple, $L=H \cap K, M=L \varphi$, and $X$ is a subgroup of $G$ containing $L$ and $M$. Then the following statements hold.

1. If $\mathcal{C}_{\cap}^{*}(G, H, K, \varphi)$ is a filtration, then $\mathcal{C}_{\cap}^{*}(X, L, M, \varphi)$ is also a filtration.

2. Let $X$ coincide with either $G$ or $K$. If $\mathcal{C}_{\cap}^{*}(G, H, K, \varphi)$ is an $(H, K)$-filtration, then $\mathcal{C}_{\cap}^{*}(X, L, M, \varphi)$ is an $(L, M)$-filtration.

Proof. By Proposition 4.1, if $N \in \mathcal{C}_{\cap}^{*}(G, H, K, \varphi)$, then $N \cap X \in \mathcal{C}_{\cap}^{*}(X, L, M, \varphi)$. Hence,

$$
\bigcap_{R \in \mathcal{C}_{\cap}^{*}(X, L, M, \varphi)} R \leqslant \bigcap_{N \in \mathcal{C}_{\cap}^{*}(G, H, K, \varphi)}(N \cap X), \quad \bigcap_{R \in \mathcal{C}_{\cap}^{*}(X, L, M, \varphi)} R L \leqslant \bigcap_{N \in \mathcal{C}_{\cap}^{*}(G, H, K, \varphi)}(N \cap X) L .
$$

If $\mathcal{C}_{\cap}^{*}(G, H, K, \varphi)$ is a filtration, then

$$
1=\bigcap_{N \in \mathcal{C}_{\cap}^{*}(G, H, K, \varphi)} N=\bigcap_{N \in \mathcal{C}_{\cap}^{*}(G, H, K, \varphi)}(N \cap X)
$$

and therefore $\mathcal{C}_{\cap}^{*}(X, L, M, \varphi)$ is also a filtration.

Let $\mathcal{C}_{\cap}^{*}(G, H, K, \varphi)$ be an $(H, K)$-filtration. Then

$$
\begin{aligned}
L \leqslant \bigcap_{R \in \mathcal{C}_{\cap}^{*}(X, L, M, \varphi)} R L \leqslant \bigcap_{N \in \mathcal{C}_{\cap}^{*}(G, H, K, \varphi)} N L=\bigcap_{N \in \mathcal{C}_{\cap}^{*}(G, H, K, \varphi)} N(H \cap K) \\
\leqslant\left(\bigcap_{N \in \mathcal{C}_{\cap}^{*}(G, H, K, \varphi)} N H\right) \cap\left(\bigcap_{N \in \mathcal{C}_{\cap}^{*}(G, H, K, \varphi)} N K\right)=H \cap K=L
\end{aligned}
$$

and therefore $\mathcal{C}_{\cap}^{*}(X, L, M, \varphi)$ is an $L$-filtration. To prove that this family is an $M$-filtration, we take an arbitrary element $x \in X \backslash M$ and indicate a subgroup $R \in \mathcal{C}_{\cap}^{*}(X, L, M, \varphi)$ satisfying the condition $x \notin M R$.

If $x \notin K$, then, because $\mathcal{C}_{\cap}^{*}(G, H, K, \varphi)$ is a $K$-filtration, there exists a subgroup $N \in \mathcal{C}_{\cap}^{*}(G, H, K, \varphi)$ such that $x \notin K N$. It follows from Proposition 4.1 and the inclusion $M(N \cap X) \leqslant K N$ that $N \cap X \in \mathcal{C}_{\cap}^{*}(X, L, M, \varphi)$ and $x \notin M(N \cap X)$. Hence, $N \cap X$ is the required subgroup.

If $x \in K$, we put $y=x \varphi^{-1}$. Since $x \notin M$, then $y \in H \backslash L$ and therefore $y \notin K$. Let us consider two cases.

Case 1. $X=G$.

Let $\mathfrak{X}=\operatorname{HNN}(G, L, M, \varphi)$. As proved above, $\mathcal{C}_{\cap}^{*}(G, L, M, \varphi)$ is an $L$-filtration. Therefore, $y \notin L R$ for some subgroup $R \in \mathcal{C}_{\cap}^{*}(G, L, M, \varphi)$. By the definition of the family $\mathcal{C}_{\cap}^{*}(G, L, M, \varphi)$, there exists a subgroup $U \in \mathcal{C}^{*}(\mathfrak{X})$ such that $R=U \cap G$. If $y \in L U$ and $y=h u$ for some $h \in L, u \in U$, then $u=h^{-1} y \in U \cap G=R$ and we get the inclusion $y \in L R$, which contradicts the choice of $R$. Therefore, $y \notin L U$ and

$$
x=y \varphi=t^{-1} y t \notin t^{-1} L U t .
$$

Since $U$ is normal in $\mathfrak{X}$, then $t^{-1} L U t=M U$. Hence, $x \notin M U$ and $x \notin M R$ because $R \leqslant U$. Thus, $R$ is the required subgroup.

Case 2. $X=K$.

Since $\mathcal{C}_{\cap}^{*}(G, H, K, \varphi)$ is a $K$-filtration, there exists a subgroup $N \in \mathcal{C}_{\cap}^{*}(G, H, K, \varphi)$ such that $y \notin K N$. By Proposition $3.4, N$ is $(H, K, \varphi)$-compatible and therefore

$$
(M(N \cap K)) \varphi^{-1}=L(N \cap H) \leqslant K N .
$$

Hence, $y \notin(M(N \cap K)) \varphi^{-1}$ and $x=y \varphi \notin M(N \cap K)$. It remains to note that, by Proposition 4.1, $N \cap K \in \mathcal{C}_{\cap}^{*}(K, L, M, \varphi)$ and thus $N \cap K$ is the required subgroup. 
Let $(G, H, K, \varphi)$ be an HNN-tuple. Following [11], we say that a subgroup $N \leqslant G$ is an ascend of a subgroup $R \leqslant K$ if $K \cap N=R$.

Let $H$ and $K$ lie in the center of $G$. We call an ascend $N \leqslant G$ of a subgroup $R \leqslant K$ canonical if $H \cap N=R \varphi^{-1}$ and $H K \cap N=\left(R \varphi^{-1}\right) R$. It should be noted that a canonical ascend $N$ is always an $(H, K, \varphi)$-compatible subgroup because $(H \cap N) \varphi=R=K \cap N$.

Proposition 4.3. If $\mathcal{C}$ is a class of groups, $(G, H, K, \varphi)$ is an $H N N$-tuple, $H$ and $K$ lie in the center of $G, L=H \cap K$, and $M=L \varphi$, then the following statements hold.

1. Suppose that $\mathcal{C}$ is closed under taking subgroups, quotient groups, and extensions, $G$ is $\mathcal{C}$-regular with respect to the subgroup $P=H K$, and $R \in \mathcal{C}^{*}(K, L, M, \varphi)$. Then there exists a canonical ascend $N \in \mathcal{C}^{*}(G, H, K, \varphi)$ of $R$. Moreover, if $X$ is a normal subgroup of $G$ such that $P \leqslant X$ and $G / X$ is residually a $\mathcal{C}$-group, then, for any finite set $S \subseteq G \backslash X$, there exists a canonical ascend $N \in \mathcal{C}^{*}(G, H, K, \varphi)$ of $R$ satisfying the condition $S \cap X N=\varnothing$.

2. If $R \in \mathcal{C}_{r+1}^{*}(K, L, M, \varphi)$ for some $r \geqslant 0$ and $N \in \mathcal{C}^{*}(G, H, K, \varphi)$ is a canonical ascend of $R$, then $N \in \mathcal{C}_{r}^{*}(G, H, K, \varphi)$.

Proof. 1. If $X=G$ and $S=\varnothing$, then $P \leqslant X, G / X$ is residually a $\mathcal{C}$-group, and $S \subseteq G \backslash X$. Therefore, it suffices to prove only the second part of Statement 1 . We put $Q=R \varphi^{-1}$ and show that $H \cap Q R=Q, K \cap Q R=R$.

Since $L, Q \leqslant H, \varphi$ is injective, and $R$ is $(L, M, \varphi)$-compatible, then

$$
L \cap Q=M \varphi^{-1} \cap R \varphi^{-1}=(M \cap R) \varphi^{-1}=L \cap R .
$$

If $g \in H \cap Q R$ and $g=x y$ for some $x \in Q, y \in R$, then $g, x \in H$,

$$
y \in H \cap R=H \cap K \cap R=L \cap R=L \cap Q,
$$

and therefore $g \in Q$. Hence, $H \cap Q R \subseteq Q$. The relation $K \cap Q R \subseteq R$ is verified in the same way. Since the opposite inclusions are obvious, the required equalities are proved.

The quotient group $P / Q R$ is an extension of $H R / Q R$ by $P / H R$. We have

$$
\begin{gathered}
H R / Q R \cong H / Q(R \cap H) \cong(H / Q) /(Q(R \cap H) / Q), \\
P / H R=H K / H R \cong K / R(H \cap K) \cong(K / R) /(R(H \cap K) / R) .
\end{gathered}
$$

Since $R \in \mathcal{C}^{*}(K, L, M, \varphi)$, then $K / R \in \mathcal{C}$ and therefore $H / Q \in \mathcal{C}$. Hence, $P / Q R \in \mathcal{C}$ because $\mathcal{C}$ is closed under taking quotient groups and extensions. Thus, using the $\mathcal{C}$-regularity of $G$ with respect to $P$, we can find a subgroup $U \in \mathcal{C}^{*}(G)$ such that $U \cap P=Q R$.

By the hypothesis of the proposition, $G / X$ is residually a $\mathcal{C}$-group and $\mathcal{C}$ is closed under taking subgroups and extensions. Hence, $\mathcal{C}$ is also closed under taking direct products of a finite number of factors and, by Proposition 3.1, there exists a subgroup $V / X \in \mathcal{C}^{*}(G / X)$ such that $S X / X \cap V / X=\varnothing$. Then $V \in \mathcal{C}^{*}(G)$ and $S \cap V=\varnothing$.

Let $N=U \cap V$. Then $S \cap X N \subseteq S \cap X V=S \cap V=\varnothing$ and, again by Proposition 3.1, $N \in \mathcal{C}^{*}(G)$. Because $P \leqslant X \leqslant V$, we have

$$
\begin{aligned}
& P \cap N=P \cap U \cap V=P \cap U=Q R, \\
& H \cap N=H \cap P \cap N=H \cap Q R=Q, \\
& K \cap N=K \cap P \cap N=K \cap Q R=R .
\end{aligned}
$$

Since $Q \varphi=R$, it follows from these equalities that $N$ is an $(H, K, \varphi)$-compatible subgroup, which is the required canonical ascend of $R$.

2. The relation $R \in \mathcal{C}_{r+1}^{*}(K, L, M, \varphi)$ means that there exists a number $n \geqslant r+3$ such that a cyclic group $C_{n}$ of order $n$ belongs to $\mathcal{C}$ and $n$ is admissible for the HNN-tuple 
$\left(K / R, L R / R, M R / R, \varphi_{R}\right)$ with the reserve $r+1$. Since $K \cap N=R$, the map of $K / R$ into $G / N$ taking a coset $k R(k \in K)$ to $k N$ is an isomorphism of $K / R$ onto the subgroup $K N / N$ of $G / N$. Under this isomorphism, the subgroups $L R / R$ and $M R / R$ are mapped onto the subgroups $L N / N$ and $M N / N$, and the isomorphism $\varphi_{R}$ corresponds to the isomorphism $\varphi_{N}$. Therefore, $n$ is admissible with the reserve $r+1$ for the HNNtuple $\left(K N / N, L N / N, M N / N, \varphi_{N}\right)$. Let us show that $H N / N \cap K N / N=L N / N$.

If $g N \in H N / N \cap K N / N$, then $g \in H N \cap K N$ and $g=h x=k y$ for some $h \in H$, $k \in K$, and $x, y \in N$. Hence, $h^{-1} k=x y^{-1} \in H K \cap N$. Since $N$ is a canonical ascend of $R$, then $H K \cap N=Q R$ (where, as above, $Q=R \varphi^{-1}$ ) and therefore $h^{-1} k=h_{1} k_{1}$ for some $h_{1} \in Q, k_{1} \in R$. Thus, $h h_{1}=k k_{1}^{-1} \in H \cap K=L$ and $h \in h_{1}^{-1} L$. Since $h_{1} \in Q \leqslant N$, then $g=h x \in L N$ and $g N \in L N / N$. Therefore, $H N / N \cap K N / N \subseteq L N / N$ and, because the opposite inclusion is obvious, the required equality is proved.

Thus, $H N / N \cap K N / N=L N / N$ and $(L N / N) \varphi_{N}=M N / N$ by the definition of $\varphi_{N}$. It follows from these equalities and Proposition 2.2 that $n$ is admissible for the HNN-tuple $\left(G / N, H N / N, K N / N, \varphi_{N}\right)$ with the reserve $r$. Since $C_{n} \in \mathcal{C}$, we have $N \in \mathcal{C}_{r}^{*}(G, H, K, \varphi)$, as required.

Let $X$ be a group, and let $Y, Z$ be its subgroups. We call a family $\Omega$ of normal subgroups of $X$ a strong $(Y, Z)$-filtration if, for any finite subset $S$ of $X$, there exists a subgroup $N \in \Omega$ such that, for each $x \in S$, the following statements hold:

1) if $x \neq 1$, then $x \notin N$;

2) if $x \notin Y$, then $x \notin Y N$;

3) if $x \notin Z$, then $x \notin Z N$.

It is easy to see that any strong $(Y, Z)$-filtration is a $(Y, Z)$-filtration and, if $\Omega$ is closed under taking finite intersections of subgroups, then any $(Y, Z)$-filtration is a strong $(Y, Z)$ filtration.

Proposition 4.4. Suppose that $\mathcal{C}$ is a class of groups closed under taking subgroups, quotient groups, and extensions, $(G, H, K, \varphi)$ is an $H N N$-tuple, $H$ and $K$ lie in the center of $G$ and are $\pi^{\prime}$-isolated in this group for some set of primes $\pi, L=H \cap K, M=L \varphi$, and $G$ is $\mathcal{C}$-regular with respect to the subgroup $P=H K$. Suppose also that there exists a normal subgroup $X$ of $G$ such that $P \leqslant X, X / P$ is a periodic $\pi^{\prime}$-group, and $G / X$ is residually a $\mathcal{C}$-group. Then the following statements hold.

1. If $\mathcal{C}^{*}(K, L, M, \varphi)$ is a strong $(L, M)$-filtration, then $\mathcal{C}^{*}(G, H, K, \varphi)$ is a strong $(H, K)$ filtration.

2. If, for some $r \geqslant 0, \mathcal{C}_{r+1}^{*}(K, L, M, \varphi)$ is a strong $(L, M)$-filtration, then $\mathcal{C}_{r}^{*}(G, H, K, \varphi)$ is a strong $(H, K)$-filtration.

Proof. We prove Statements 1 and 2 simultaneously. Suppose that $S$ is a finite subset of $G$ and $g \in S$. Then:

1) if $g \in K \backslash H$, then $g \notin L$;

2) if $g \in H \backslash K$, then $g \varphi \notin M$;

3) if $g \in P \backslash(H \cup K)$ and $g=h k$, where $h \in H, k \in K$, then $k \notin L, h \varphi \notin M$;

4) if $g \in X \backslash P, q$ is the order of $g$ modulo $P$, and $g^{q}=h k$, where $h \in H, k \in K$, then $k \notin L, h \varphi \notin M$.

Indeed, if $g \in K \backslash H$, then $g \notin H \cap K=L$; if $g \in H \backslash K$, then $g \notin H \cap K=L$ and therefore $g \varphi \notin M$. If $g \in P \backslash(H \cup K)$ and $g=h k$ for some $h \in H, k \in K$, then $h \notin K, k \notin H$, and, as above, $k \notin L, h \varphi \notin M$. Suppose that $g \in X \backslash P, q$ is the order of $g$ modulo $P$, and $g^{q}=h k$, where $h \in H, k \in K$. Since $g \notin H \cup K, H$ and $K$ are $\pi^{\prime}$-isolated in $G$, and, by the hypothesis of the proposition, $q$ is a $\pi^{\prime}$-number, then $h k \notin H \cup K$. Hence, using the above argument, we get $k \notin L, h \varphi \notin M$. 
Thus, because the family $\mathcal{C}^{*}(K, L, M, \varphi)$ or $\mathcal{C}_{r+1}^{*}(K, L, M, \varphi)$ is a strong $(L, M)$-filtration, we can choose a subgroup $R$ from this family so that, for each $g \in S$, the following conditions hold:

1) if $g \in L$ and $g \neq 1$, then $g \notin R$;

2) if $g \in K \backslash H$, then $g \notin L R$;

3) if $g \in H \backslash K$, then $g \varphi \notin M R$;

4) if $g \in P \backslash(H \cup K)$ and $g=h k$, where $h \in H, k \in K$, then $k \notin L R, h \varphi \notin M R$;

5) if $g \in X \backslash P, q$ is the order of $g$ modulo $P$, and $g^{q}=h k$, where $h \in H, k \in K$, then $k \notin L R, h \varphi \notin M R$.

It is sufficient for us that, for each $g \in S$, the fourth and fifth conditions hold for some fixed choice of $h$ and $k$. However, if $h_{1} k_{1}=h_{2} k_{2}$ for some $h_{1}, h_{2} \in H, k_{1}, k_{2} \in K$, then $h_{1}^{-1} h_{2}=k_{1} k_{2}^{-1} \in L$. Therefore, if $k_{1} \notin L R$ and $h_{1} \varphi \notin M R$, then $k_{2} \notin L R$ and $h_{2} \varphi \notin M R$.

Let $S_{1}=S \backslash X$, and let $Q=R \varphi^{-1}$. By Proposition 4.3, there exists a canonical ascend $N \in \mathcal{C}^{*}(G, H, K, \varphi)$ of $R$ such that $S_{1} \cap X N=\varnothing$ and, if $R \in \mathcal{C}_{r+1}^{*}(K, L, M, \varphi)$, then $N \in \mathcal{C}_{r}^{*}(G, H, K, \varphi)$. To complete the proof, it suffices to show that, for each $g \in S$, the following statements hold:

1) if $g \neq 1$, then $g \notin N$;

2) if $g \notin H$, then $g \notin H N$;

3) if $g \notin K$, then $g \notin K N$.

Let us consider several cases.

Case 1: $g \in L$ and $g \neq 1$.

Since $R=N \cap K, g \in K$, and $g \notin R$ by the choice of $R$, then $g \notin N$.

Case 2: $g \in K \backslash H$.

If $g \in H N$ and $g=h x$ for some $h \in H, x \in N$, then $x=h^{-1} g \in N \cap P$. Since $N$ is a canonical ascend of $R$, then $N \cap P=Q R$ and $x=h_{1} k$ for some $h_{1} \in Q, k \in R$. Hence, $g k^{-1}=h h_{1} \in H \cap K=L, g \in L R$, and we get a contradiction with the choice of $R$.

Case 3: $g \in H \backslash K$.

As in the previous case, if $g \in K N$ and $g=k x$ for some $k \in K, x \in N$, then $x=k^{-1} g \in N \cap P=R Q$ and therefore $x=k_{1} h$ for some $h \in Q, k_{1} \in R$. Hence, $g h^{-1}=k k_{1} \in L, g=\left(k k_{1}\right) h \in L Q, g \varphi \in M R$, and we again get a contradiction with the choice of $R$.

Case 4: $g \in P \backslash(H \cup K)$.

Let $g=h k$, where $h \in H, k \in K$. If $g \in H N$ and $g=h_{1} x$ for some $h_{1} \in H, x \in N$, then it follows from the equality $h k=h_{1} x$ that $x \in P$ and therefore $x \in N \cap P=Q R$. Hence, $x=h_{2} k_{2}$ for some $h_{2} \in Q, k_{2} \in R, h k=h_{1} h_{2} k_{2}$, and $k k_{2}^{-1}=h^{-1} h_{1} h_{2} \in L$. Thus, we get the relations $k=\left(h^{-1} h_{1} h_{2}\right) k_{2} \in L R$, which contradict the choice of $R$.

Similarly, if $g \in K N$ and $g=k_{1} x$ for some $k_{1} \in K, x \in N$, then again $x \in N \cap P=Q R$ and $x=h_{2} k_{2}$ for some $h_{2} \in Q, k_{2} \in R$. Hence, $h k=k_{1} h_{2} k_{2}$ and, since $[H, K]=1$, then $h h_{2}^{-1}=k^{-1} k_{1} k_{2} \in L, h=\left(k^{-1} k_{1} k_{2}\right) h_{2} \in L Q$, and $h \varphi \in M R$. The last inclusion again contradicts the choice of $R$.

Case 5: $g \in X \backslash P$.

As already proved above, it follows from the relation $g \in X \backslash P$ that $g^{q} \notin H \cup K$, where $q$ is the order of $g$ modulo $P$. Hence, using the same argument as in Case 4, we get $g^{q} \notin H N \cup K N$. Then $g \notin H N \cup K N$, as required.

Case 6: $g \notin X$.

Since $g \notin X$, then $g \in S_{1}, g \notin X N$ by the choice of $N$, and $g \notin H N \cup K N$ because $H \cup K \subseteq X$. 


\section{NeCESSARY AND SUfficient CONDitions for the $\mathcal{C}$-Residuality OF HNN-EXTENSIONS}

Throughout this section, it is assumed that $(G, H, K, \varphi)$ is an HNN-tuple and $\mathfrak{G}=$ $\operatorname{HNN}(G, H, K, \varphi)$. Let us recall that any element $g \in \mathfrak{G}$ can be written in a reduced form:

$$
g=g_{0} t^{\varepsilon_{1}} g_{1} \ldots t^{\varepsilon_{n}} g_{n}
$$

where $g_{i} \in G, \varepsilon_{i}= \pm 1$, and if $-\varepsilon_{i}=1=\varepsilon_{i+1}$, then $g_{i} \notin H$, if $\varepsilon_{i}=1=-\varepsilon_{i+1}$, then $g_{i} \notin K$. Britton's lemma (see, e. g., [9, Ch. IV, Section 2]) says that an element of $\mathfrak{G}$ is non-trivial if it has a reduced form containing at least one letter $t$ or $t^{-1}$. As a consequence, all the reduced forms of an element $g \in \mathfrak{G}$ have the same number of occurrences of the letters $t$ and $t^{-1}$. This number is called the length of $g$ and is denoted in this paper by $|g|$.

Proposition 5.1. If $\mathcal{C}$ is a root class of groups, then the following statements hold.

1. Every free group is residually a $\mathcal{C}$-group [3, Theorem 1$]$.

2. Any extension of a residually $\mathcal{C}$-group by a $\mathcal{C}$-group is residually a $\mathcal{C}$-group [7, Lemma 1.5].

The following proposition is a generalization of Theorem 4.2 from [4] and is partially proved in [29].

Proposition 5.2. Let $\mathcal{C}$ be a class of groups.

1. If $\mathfrak{G}$ is residually a $\mathcal{C}$-group, then $\mathcal{C}_{\cap}^{*}(G, H, K, \varphi)$ is a filtration.

2. If $\mathfrak{G}$ is residually a $\mathcal{C}$-group, $H$ and $K$ are proper central subgroups of $G$, then $\mathcal{C}_{\cap}^{*}(G, H, K, \varphi)$ is an $(H, K)$-filtration.

3. If $\mathcal{C}$ is a root class of groups and $\mathcal{C}_{\cap}^{*}(G, H, K, \varphi)$ is an $(H, K)$-filtration, then $\mathfrak{G}$ is residually a $\mathcal{C}$-group.

Proof. 1. Since $\mathfrak{G}$ is residually a $\mathcal{C}$-group, then

$$
1=\bigcap_{U \in \mathcal{C}^{*}(\mathfrak{G})} U=\bigcap_{U \in \mathcal{C}^{*}(\mathfrak{G})}(U \cap G)=\bigcap_{N \in \mathcal{C}_{\cap}^{*}(G, H, K, \varphi)} N
$$

as required.

2. Suppose that $\mathcal{C}_{\cap}^{*}(G, H, K, \varphi)$ is not an $H$-filtration and

$$
g_{1} \in\left(\bigcap_{N \in \mathcal{C}_{\cap}^{*}(G, H, K, \varphi)} H N\right) \backslash H .
$$

Because $K \neq G$, we can take an element $g_{2} \in G \backslash K$ and put $g=\left[t^{-1} g_{1} t, g_{2}\right]$. Then $|g|=4$ and therefore $g \neq 1$. Since $\mathfrak{G}$ is residually a $\mathcal{C}$-group, there exists a subgroup $U \in \mathcal{C}^{*}(\mathfrak{G})$ such that $g \notin U$. By the choice of $g_{1}$, the inclusion $g_{1} \in H(U \cap G)$ holds, and therefore $g_{1} \equiv h(\bmod U)$ for some $h \in H$. Hence,

$$
g \equiv\left[t^{-1} h t, g_{2}\right]=\left[h \varphi, g_{2}\right] \quad(\bmod U) .
$$

Since $h \varphi \in K$ and $K$ is central in $G$, then $\left[h \varphi, g_{2}\right]=1$, and we get a contradiction with the choice of $U$. The fact that $\mathcal{C}_{\cap}^{*}(G, H, K, \varphi)$ is a $K$-filtration is proved in a similar way.

3. Let $g \in \mathfrak{G} \backslash\{1\}$. We show that there exists a subgroup $N \in \mathcal{C}_{\cap}^{*}(G, H, K, \varphi)$ such that $g \rho_{N} \neq 1$.

If $g \in G$, then, because $\mathcal{C}_{\cap}^{*}(G, H, K, \varphi)$ is a filtration, $g$ does not belong to some subgroup $N \in \mathcal{C}_{\cap}^{*}(G, H, K, \varphi)$. Since $\rho_{N}$ extends the natural homomorphism $G \rightarrow G / N$, then $g \rho_{N} \neq 1$ and therefore $N$ is the required subgroup.

Suppose that $g \notin G$ and $g=g_{0} t^{\varepsilon_{1}} g_{1} \ldots t^{\varepsilon_{n}} g_{n}$, where $g_{i} \in G, \varepsilon_{i}= \pm 1$, and if $-\varepsilon_{i}=1=\varepsilon_{i+1}$, then $g_{i} \notin H$, if $\varepsilon_{i}=1=-\varepsilon_{i+1}$, then $g_{i} \notin K$. It should be noted that, since $g \notin G$, then $n \geqslant 1$. For each $i \in\{0,1, \ldots, n\}$, we define a subgroup $N_{i} \in \mathcal{C}_{\cap}^{*}(G, H, K, \varphi)$ as follows. 
If $1 \leqslant i \leqslant n-1$ and $-\varepsilon_{i}=1=\varepsilon_{i+1}$, then $g_{i} \notin H$ and, since $\mathcal{C}_{\cap}^{*}(G, H, K, \varphi)$ is an $(H, K)$-filtration, we can find a subgroup $N_{i} \in \mathcal{C}_{\cap}^{*}(G, H, K, \varphi)$ such that $g_{i} \notin H N_{i}$. Similarly, if $1 \leqslant i \leqslant n-1$ and $\varepsilon_{i}=1=-\varepsilon_{i+1}$, we choose a subgroup $N_{i} \in \mathcal{C}_{\cap}^{*}(G, H, K, \varphi)$ so that $g_{i} \notin K N_{i}$. For all other $i \in\{0,1, \ldots, n\}$, we put $N_{i}=G$. The last choice is possible because $\mathcal{C}$ is root and therefore the trivial group belongs to $\mathcal{C}, \mathfrak{G} \in \mathcal{C}^{*}(\mathfrak{G})$, and $G \in \mathcal{C}_{\cap}^{*}(G, H, K, \varphi)$.

Let

$$
N=\bigcap_{i=0}^{n} N_{i} .
$$

Then $N \in \mathcal{C}_{\cap}^{*}(G, H, K, \varphi)$ by Proposition 3.2 and, for any $i \in\{1,2, \ldots, n-1\}$, if $-\varepsilon_{i}=1=\varepsilon_{i+1}$, then $g_{i} \notin H N$, if $\varepsilon_{i}=1=-\varepsilon_{i+1}$, then $g_{i} \notin K N$. Hence,

$$
g \rho_{N}=\left(g_{0} N\right) t^{\varepsilon_{1}}\left(g_{1} N\right) \ldots t^{\varepsilon_{n}}\left(g_{n} N\right)
$$

is a reduced form of $g \rho_{N}$ of length $n \geqslant 1$ and, in particular, $g \rho_{N} \neq 1$. Thus, $N$ is the required subgroup.

Let us now show that $\mathfrak{G}_{N}=\operatorname{HNN}\left(G / N, H N / N, K N / N, \varphi_{N}\right)$ is residually a $\mathcal{C}$-group and therefore $\rho_{N}$ can be extended to a homomorphism of $\mathfrak{G}$ onto a group from $\mathcal{C}$ taking $g$ to a non-trivial element. Suppose that $U \in \mathcal{C}^{*}(\mathfrak{G})$ is a subgroup satisfying the equality $N=U \cap G$ and $\sigma: \mathfrak{G}_{N} \rightarrow \mathfrak{G} / U$ is the mapping defined by the rule: $\left(x \rho_{N}\right) \sigma=x U(x \in \mathfrak{G})$. Since the kernel of $\rho_{N}$ coincides with the normal closure of $N$ in $\mathfrak{G}$, it is contained in $U$ and therefore $\sigma$ is well defined. It is also easy to see that $\sigma$ is a surjective homomorphism. Since $G \rho_{N}=G / N$ and $N=U \cap G$, then $\operatorname{ker} \sigma \cap G / N=1$. Hence, $\operatorname{ker} \sigma$ is free (see, e. g., [5]) and $\mathfrak{G}_{N}$ is an extension of the free group $\operatorname{ker} \sigma$ by the $\mathcal{C}$-group $\mathfrak{G} / U$. Such an extension is residually a $\mathcal{C}$-group by Proposition 5.1 .

Proposition 5.3. Suppose that $\mathcal{C}$ is a class of groups consisting only of periodic groups, $H$ is a central subgroup of $G$, and $H \leqslant K \neq G$. If $\mathfrak{G}$ is residually a $\mathcal{C}$-group, then $H=K$.

Proof. Suppose that $H \neq K$ and $k \in K \backslash H$. Suppose also that $g \in G \backslash K$ and $x=\left[t^{-1} k t, g\right]$. Then $|x|=4$ and therefore $x \neq 1$. Since $\mathfrak{G}$ is residually a $\mathcal{C}$-group, there exists a subgroup $U \in \mathcal{C}^{*}(\mathfrak{G})$ such that $x \notin U$. Because $\mathcal{C}$ consists only of periodic groups, $t$ has a finite order modulo $U$ and therefore $t^{-1} \equiv t^{m}(\bmod U)$ for some $m>0$. It follows from the last relation and the inclusion $H \leqslant K$ that

$$
t^{-1} k t \equiv t^{m} k t^{-m}=k \varphi^{-m} \in H \quad(\bmod U) .
$$

Since $H$ is central in $G$, then $\left[k \varphi^{-m}, g\right]=1$. Thus, $x \in U$, and we get a contradiction with the choice of $U$.

Proposition 5.4. Suppose that $\mathcal{C}$ is a class of groups closed under taking subgroups and direct products of a finite number of factors. Suppose also that $G$ is residually a $\mathcal{C}$-group and there exists a subgroup $Q \in \mathcal{C}^{*}(G)$ satisfying at least one of the following conditions:

( $\alpha) H \cap Q=1=K \cap Q$,

( $\beta) Q \leqslant H \cap K$ and $Q \varphi=Q$.

Then $H$ and $K$ are $\mathcal{C}$-separable in $G$.

Proof. We take an element $g \in G \backslash H$ and find a subgroup $N \in \mathcal{C}^{*}(G)$ such that $g \notin H N$.

If $Q \leqslant H$ or $g \notin H Q$, then $Q$ is the required subgroup. Therefore, we can assume that $H \cap Q=1$ and $g=h x$ for some $h \in H, x \in Q$. Since $g \notin H$, then $x \neq 1$ and, because $G$ is residually a $\mathcal{C}$-group, there exists a subgroup $M \in \mathcal{C}^{*}(G)$ that does not contain $x$. We put $N=M \cap Q$. Then $N \in \mathcal{C}^{*}(G)$ by Proposition 3.1. If $g=h_{1} x_{1}$ for some $h_{1} \in H, x_{1} \in N$, then $x x_{1}^{-1}=h^{-1} h_{1} \in H \cap Q=1$. Hence, $x=x_{1} \in N \leqslant M$, and we get a contradiction with the choice of $M$. Therefore, $g \notin H N$ and $N$ is the required subgroup.

Thus, $H$ is $\mathcal{C}$-separable in $G$. The $\mathcal{C}$-separability of $K$ is proved in a similar way. 
Proposition 5.5. Suppose that $\mathcal{C}$ is a root class of groups containing infinite groups, $G$ is residually a $\mathcal{C}$-group, $H$ and $K$ are central subgroups of $G$. Suppose also that there exists a subgroup $Q \in \mathcal{C}^{*}(G)$ satisfying at least one of the following conditions:

( $\alpha) H \cap Q=1=K \cap Q$,

( $\beta) Q \leqslant H \cap K$ and $Q \varphi=Q$.

Then $\mathcal{C}^{*}(G, H, K, \varphi)$ is an $(H, K)$-filtration.

Proof. We only need to show that every subgroup from $\mathcal{C}^{*}(G)$ contains some subgroup from $\mathcal{C}^{*}(G, H, K, \varphi)$. Since $G$ is residually a $\mathcal{C}$-group and $H, K$ are $\mathcal{C}$-separable in $G$ by Proposition 5.4, then it will follow from this fact that $\mathcal{C}^{*}(G, H, K, \varphi)$ is an $(H, K)$-filtration.

So, let $L \in \mathcal{C}^{*}(G)$. By Proposition 3.1, the subgroup $M=L \cap Q$ belongs to $\mathcal{C}^{*}(G)$. If $(\alpha)$ holds, then

$$
(H \cap M) \varphi=(H \cap L \cap Q) \varphi=1=K \cap L \cap Q=K \cap M
$$

and therefore $M \in \mathcal{C}^{*}(G, H, K, \varphi)$. If $(\beta)$ holds, then the subgroup

$$
N=\bigcap_{i \in \mathbb{Z}} M \varphi^{i}
$$

belongs to the same family.

Indeed, $N$ lies in the center of $G$, is $\varphi$-invariant, and therefore is $(H, K, \varphi)$-compatible. Since $Q / M \leqslant G / M$ and $\mathcal{C}$ is closed under taking subgroups, then $M \in \mathcal{C}^{*}(Q)$. Because $Q$ is $\varphi$-invariant, the restriction of $\varphi$ to this subgroup is its automorphism and therefore $M \varphi^{i} \in \mathcal{C}^{*}(Q)$. Hence, $Q / N$ is embedded into the Cartesian product of countably many isomorphic $\mathcal{C}$-groups $Q / M \varphi^{i}$. Since $\mathcal{C}$ is root and contains infinite groups, then it also contains the indicated Cartesian product, its subgroup $Q / N$, and the group $G / N$, which is an extension of $Q / N$ by the $\mathcal{C}$-group $G / Q$. Therefore, $N \in \mathcal{C}^{*}(G)$.

Proposition 5.6. Suppose that $\mathcal{C}$ is a class of groups closed under taking subgroups and direct products of a finite number of factors, $H$ and $K$ are proper central subgroups of finite index of $G$. If $\mathfrak{G}$ is residually a $\mathcal{C}$-group, then there exists a subgroup $Q \in \mathcal{C}^{*}(G)$ such that $Q \leqslant H \cap K$ and $Q \varphi=Q$.

Proof. Because $H$ and $K$ are of finite index in $G$, the subgroup $H \cap K$ has the same property. Let $1=g_{1}, g_{2}, \ldots, g_{n}$ be a complete set of cosets representatives of this subgroup in $G$, and let $S=\left\{g_{2}, \ldots, g_{n}\right\}$. Since $\mathfrak{G}$ is residually a $\mathcal{C}$-group, then, by Proposition 5.2 , $\mathcal{C}_{\cap}^{*}(G, H, K, \varphi)$ is an $(H, K)$-filtration. Hence,

$$
H \cap K \leqslant \bigcap_{N \in \mathcal{C}_{\cap}^{*}(G, H, K, \varphi)}(H \cap K) N \leqslant\left(\bigcap_{N \in \mathcal{C}_{\cap}^{*}(G, H, K, \varphi)} H N\right) \cap\left(\bigcap_{N \in \mathcal{C}_{\cap}^{*}(G, H, K, \varphi)} K N\right)=H \cap K
$$

and therefore, for each $s \in S$, there exists a subgroup $Q_{s} \in \mathcal{C}_{\cap}^{*}(G, H, K, \varphi)$ such that $s \notin(H \cap K) Q_{s}$. Let us show that

$$
Q=\bigcap_{s \in S} Q_{s}
$$

is the required subgroup.

Indeed, if $g \in G \backslash(H \cap K)$ and $g=x s$ for suitable $s \in S, x \in H \cap K$, then $x^{-1} g=s \notin(H \cap K) Q_{s}$ and therefore $x^{-1} g \notin(H \cap K) Q$. Hence, $g \notin(H \cap K) Q$ and, because $g$ is chosen arbitrarily, $(H \cap K) Q \leqslant H \cap K$. Thus, $Q \leqslant H \cap K$. It remains to note that $Q \in \mathcal{C}_{\cap}^{*}(G, H, K, \varphi)$ by Proposition 3.2 and $\mathcal{C}_{\cap}^{*}(G, H, K, \varphi) \subseteq \mathcal{C}^{*}(G, H, K, \varphi)$ by Proposition 3.4. Therefore, $Q \in \mathcal{C}^{*}(G)$ and $Q \varphi=(Q \cap H) \varphi=Q \cap K=Q$. 
We conclude this section with two criteria for the root-class residuality of split extensions.

Proposition 5.7. Suppose that $\mathcal{C}$ is a root class of groups consisting only of periodic groups and closed under taking quotient groups. Suppose also that $G$ is an abelian group, $H=G=K$, and $\Omega$ is the family of subgroups of $G$ defined as follows: $N \in \Omega$ if and only if $N \in \mathcal{C}^{*}(G), N \varphi=N$, and the automorphism $\varphi_{N}$ of the group $G / N$ induced by the automorphism $\varphi$ has a finite order, which is a $\pi(\mathcal{C})$-number. Then $\mathfrak{G}$ is residually a $\mathcal{C}$-group if and only if $\bigcap_{N \in \Omega} N=1$.

Proof. Since $H=G=K$, then any filtration is an $(H, K)$-filtration. Therefore, by Proposition $5.2, \mathfrak{G}$ is residually a $\mathcal{C}$-group if and only if $\mathcal{C}_{\cap}^{*}(G, H, K, \varphi)$ is a filtration. It remains to note that, by Proposition 3.5, a subgroup $N$ belongs to $\mathcal{C}_{\cap}^{*}(G, H, K, \varphi)$ if and only if $N \varphi=N, G / N \in \mathcal{C}$, and the order of $\varphi_{N}$ is finite and is a $\pi(\mathcal{C})$-number.

Proposition 5.8. Suppose that $\mathcal{C}$ is a root class of groups consisting only of period$i c$ groups and closed under taking quotient groups, $G$ is an abelian residually $\mathcal{C}$-group, and $H=G=K$. If the order $q$ of the automorphism $\varphi$ is finite, then $\mathfrak{G}$ is residually a $\mathcal{C}$-group if and only if $q$ is a $\pi(\mathcal{C})$-number.

Proof. Necessity. Because $q$ is the order of $\varphi$, for any $i \in\{1, \ldots, q-1\}$, there exists an element $g_{i} \in G$ such that $g_{i} \varphi^{i} \neq g_{i}$. Let

$$
S=\left\{g_{i} \varphi^{i} g_{i}^{-1} \mid 1 \leqslant i \leqslant q-1\right\} .
$$

Since $\mathfrak{G}$ is residually a $\mathcal{C}$-group, then, by Proposition 3.1, there exists a subgroup $U \in \mathcal{C}^{*}(\mathfrak{G})$ such that $U \cap S=\varnothing$. If $N=U \cap G$, then $N \in \mathcal{C}_{\cap}^{*}(G, H, K, \varphi)$ and, by Proposition 3.5, $N$ is $\varphi$-invariant, the automorphism $\varphi_{N}$ of $G / N$ induced by $\varphi$ has a finite order $q_{N}$, and this order is a $\pi(\mathcal{C})$-number. It remains to note that $N \cap S=\varnothing$ and therefore $q_{N}=q$.

Sufficiency. Suppose that $\Omega$ is the family of subgroups defined in Proposition 5.7, $M \in \mathcal{C}^{*}(G)$, and

$$
N=\bigcap_{i=0}^{q-1} M \varphi^{i} .
$$

Then $N \leqslant M, N \varphi=N$, and, by Proposition 3.1, $N \in \mathcal{C}^{*}(G)$. The order of the automorphism $\varphi_{N}$ induced by $\varphi$ divides $q$ and therefore is a $\pi(\mathcal{C})$-number. Hence, $N \in \Omega$.

Thus, any subgroup from $\mathcal{C}^{*}(G)$ contains a subgroup from $\Omega$ and, since $G$ is residually a $\mathcal{C}$-group, $\Omega$ is a filtration. Therefore, $\mathfrak{G}$ is residually a $\mathcal{C}$-group by Proposition 5.7.

\section{Proofs of Theorems 1-4 and Corollaries 1-4}

Throughout this section, it is assumed that $(G, H, K, \varphi)$ is an HNN-tuple, $H$ and $K$ lie in the center of $G$, and $\mathfrak{G}=\operatorname{HNN}(G, H, K, \varphi)$. It is also assumed that

$$
K_{0}=G, H_{1}=H, K_{1}=K, H_{i+1}=H_{i} \cap K_{i}, K_{i+1}=H_{i+1} \varphi, P_{i}=H_{i} K_{i}(i \geqslant 1) .
$$

Proposition 6.1. Suppose that $\mathcal{C}$ is a root class of groups and $H \neq G \neq K$. If $\mathfrak{G}$ is residually a $\mathcal{C}$-group, then, for any $n \geqslant 1$, the $H N N$-extensions $\mathfrak{G}_{n-1}=\operatorname{HNN}\left(G, H_{n}, K_{n}, \varphi\right)$ and $\mathfrak{K}_{n-1}=\operatorname{HNN}\left(K_{n-1}, H_{n}, K_{n}, \varphi\right)$ are also residually $\mathcal{C}$-groups.

Proof. Since $\mathfrak{G}$ is residually a $\mathcal{C}$-group, then the family $\mathcal{C}_{\cap}^{*}(G, H, K, \varphi)$ is an $(H, K)$-filtration by Proposition 5.2. Repeatedly using Proposition 4.2, we see that $\mathcal{C}_{\cap}^{*}\left(G, H_{n}, K_{n}, \varphi\right)$ and $\mathcal{C}_{\cap}^{*}\left(K_{n-1}, H_{n}, K_{n}, \varphi\right)$ are $\left(H_{n}, K_{n}\right)$-filtrations, and, again by Proposition $5.2, \mathfrak{G}_{n-1}$ and $\mathfrak{K}_{n-1}$ are residually $\mathcal{C}$-groups. 
Proposition 6.2. The following statements hold.

1. Let $\pi$ be a set of primes. If $H_{n}$ and $K_{n}$ are $\pi^{\prime}$-isolated in $G$ for some $n \geqslant 1$, then $H_{i}$ and $K_{i}$ are $\pi^{\prime}$-isolated in this group for all $i \geqslant n$.

2. If a number $n \geqslant 1$ and a subgroup $Q$ are such that $Q \leqslant H_{n} \cap K_{n}$ and $Q \varphi=Q$, then $Q \leqslant H_{i} \cap K_{i}$ for all $i \geqslant n$.

3. Let $\mathcal{C}$ be a class of groups closed under taking subgroups and extensions. If $H \in \mathcal{C}^{*}(G)$ and $K \in \mathcal{C}^{*}(G)$, then $H_{i} \in \mathcal{C}^{*}(G)$ and $K_{i} \in \mathcal{C}^{*}(G)$ for all $i \geqslant 1$.

Proof. Let us use induction on $i$ and note that, for all three statements, the induction base is obvious.

1. Suppose that $H_{i}$ and $K_{i}$ are $\pi^{\prime}$-isolated in $G$ for some $i \geqslant n$. If an element $g \in G$ and a number $q \in \pi^{\prime}$ are such that $g^{q} \in H_{i+1}$, then $g^{q} \in H_{i}$ and $g^{q} \in K_{i}$. Since $H_{i}$ and $K_{i}$ are $\pi^{\prime}$-isolated, then $g \in H_{i} \cap K_{i}=H_{i+1}$ and therefore $H_{i+1}$ is $\pi^{\prime}$-isolated in $G$. If, for some $g \in G$ and $q \in \pi^{\prime}$, the inclusion $g^{q} \in K_{i+1}$ holds, then $g \in K_{i}$ because $K_{i}$ is $\pi^{\prime}$-isolated. Hence, the element $g \varphi^{-1}$ is defined and $\left(g \varphi^{-1}\right)^{q} \in H_{i+1}$. Since $H_{i+1}$ is $\pi^{\prime}$-isolated, it follows from the last inclusion that $g \varphi^{-1} \in H_{i+1}$ and $g \in K_{i+1}$. Thus, $K_{i+1}$ is also $\pi^{\prime}$-isolated in $G$.

2. Let $Q \leqslant H_{i} \cap K_{i}$ for some $i \geqslant n$. Then

$$
Q \leqslant H_{i+1}=H_{i} \cap K_{i}, \quad Q \varphi \leqslant H_{i+1} \varphi=K_{i+1},
$$

and since $Q \varphi=Q$, then $Q \leqslant H_{i+1} \cap K_{i+1}$.

3. Let $\mathcal{C}^{*}(G)$ contain $H_{i}$ and $K_{i}$ for some $i \geqslant 1$. Then $H_{i+1}=H_{i} \cap K_{i} \in \mathcal{C}^{*}(G)$ by Proposition 3.1. Since $\mathcal{C}$ is closed under taking subgroups, then $H_{i+1} \in \mathcal{C}^{*}\left(H_{i}\right)$ and it follows from the equalities $H_{i} \varphi=K_{i}, H_{i+1} \varphi=K_{i+1}$ that $K_{i+1} \in \mathcal{C}^{*}\left(K_{i}\right)$. Hence, the quotient group $G / K_{i+1}$ is an extension of the $\mathcal{C}$-group $K_{i} / K_{i+1}$ by the $\mathcal{C}$-group $G / K_{i}$, and $K_{i+1} \in \mathcal{C}^{*}(G)$ because $\mathcal{C}$ is closed under taking extensions.

Proposition 6.3. [22, Proposition 5] Suppose that $\mathcal{C}$ is a class of groups consisting only of periodic groups, $X$ is a group, and $Y$ is a subgroup of $X$. If $Y$ is $\mathcal{C}$-separable in $X$, then it is $\pi(\mathcal{C})^{\prime}$-isolated in this group. In particular, if $X$ is residually a $\mathcal{C}$-group, then it has no $\pi(\mathcal{C})^{\prime}$-torsion.

If $\pi$ is a set of primes, $X$ is a group, and $Y$ is a subgroup of $X$, then we denote by $\mathcal{R}_{\pi^{\prime}}(X, Y)$ the set of elements of $X$ defined as follows: $x \in \mathcal{R}_{\pi^{\prime}}(X, Y)$ if and only if $x^{q} \in Y$ for some $\pi^{\prime}$-number $q$.

Obviously, $\mathcal{R}_{\pi^{\prime}}(X, Y) \subseteq \mathcal{I}_{\pi^{\prime}}(X, Y)$ and the equality $\mathcal{I}_{\pi^{\prime}}(X, Y)=\mathcal{R}_{\pi^{\prime}}(X, Y)$ holds if and only if $\mathcal{R}_{\pi^{\prime}}(X, Y)$ is a subgroup. It is easy to see that, if $X$ is an abelian group, then $\mathcal{R}_{\pi^{\prime}}(X, Y)$ is always a subgroup.

Proposition 6.4. Suppose that $\mathcal{C}$ is a class of groups closed under taking quotient groups, $X$ is residually a $\mathcal{C}$-group, $Y$ is a central subgroup of $X$. Then the $\pi(\mathcal{C})^{\prime}$-isolator $\mathcal{I}_{\pi(\mathcal{C})^{\prime}}(X, Y)$ is contained in the center of $X$ and coincides with the set $\mathcal{R}_{\pi(\mathcal{C})^{\prime}}(X, Y)$.

Proof. Let $Z$ be the center of $X$. We show that $Z$ is $\mathcal{C}$-separable in $X$.

If $x \in X \backslash Z$, then $[x, y] \neq 1$ for some $y \in X$ and, because $X$ is residually a $\mathcal{C}$-group, there exists a subgroup $N \in \mathcal{C}^{*}(X)$ such that $[x, y] \notin N$. Hence, $[x N, y N] \neq N$ and therefore $x N$ does not belong to the center $\mathcal{Z}(X / N)$ of $X / N$. It is easy to see that $Z N / N \leqslant \mathcal{Z}(X / N)$. Thus, $x N \notin Z N / N$ and $x \notin Z N$. It remains to note that $X / Z N \cong(X / N) /(Z N / N) \in \mathcal{C}$ because $\mathcal{C}$ is closed under taking quotient groups.

Now, it follows from Proposition 6.3 that $Z$ is $\pi(\mathcal{C})^{\prime}$-isolated in $X$, and since $Y \leqslant Z$, then $\mathcal{I}_{\pi(\mathcal{C})^{\prime}}(X, Y) \leqslant Z$. Hence, $\mathcal{I}_{\pi(\mathcal{C})^{\prime}}(X, Y)=\mathcal{I}_{\pi(\mathcal{C})^{\prime}}(Z, Y), \mathcal{R}_{\pi(\mathcal{C})^{\prime}}(X, Y)=\mathcal{R}_{\pi(\mathcal{C})^{\prime}}(Z, Y)$, and $\mathcal{I}_{\pi(\mathcal{C})^{\prime}}(Z, Y)=\mathcal{R}_{\pi(\mathcal{C})^{\prime}}(Z, Y)$ by the above remark. 
Proposition 6.5. [25, Proposition 3] Suppose that $\mathcal{C}$ is a class of groups closed under taking quotient groups, $X$ is a group, and $Y$ is a normal subgroup of $X$. Then $Y$ is $\mathcal{C}$-separable in $X$ if and only if $X / Y$ is residually a $\mathcal{C}$-group.

Proof of Theorem 3. Necessity. Since $\mathfrak{G}$ is residually a $\mathcal{C}$-group and $\mathcal{C}$ is closed under taking subgroups, then $E$ is residually a $\mathcal{C}$-group. By Proposition 6.1, the HNN-extension $\mathfrak{G}_{n-1}=\operatorname{HNN}\left(G, H_{n}, K_{n}, \varphi\right)$ is also residually a $\mathcal{C}$-group. Since $H_{n}=H_{n+1}=H_{n} \cap K_{n}$, then $H_{n} \leqslant K_{n}$, and we get the equality $H_{n}=K_{n}$ by applying Proposition 5.3 to $\mathfrak{G}_{n-1}$.

It follows from Proposition 5.2 that $\mathcal{C}_{\cap}^{*}(G, H, K, \varphi)$ is an $(H, K)$-filtration. By Proposition 3.4, $\mathcal{C}_{\cap}^{*}(G, H, K, \varphi) \subseteq \mathcal{C}^{*}(G)$, and therefore $\mathcal{C}^{*}(G)$ is also an $(H, K)$-filtration. Hence, $H$ and $K$ are $\mathcal{C}$-separable in $G$ and $\pi(\mathcal{C})^{\prime}$-isolated in this group by Proposition 6.3.

Sufficiency. Since

$$
H_{n+1}=H_{n}=K_{n}, \quad K_{n+1}=H_{n+1} \varphi=H_{n} \varphi=K_{n},
$$

then $\mathfrak{K}_{n}=\operatorname{HNN}\left(K_{n}, H_{n+1}, K_{n+1}, \varphi\right)$ is a split extension of $K_{n}$ by the infinite cyclic group generated by $t$. Therefore, the mapping of the generators of $\mathfrak{K}_{n}$ to the corresponding elements of $\mathfrak{G}$ can be extended to an injective homomorphism taking $\mathfrak{K}_{n}$ onto $E$. Hence, $\mathfrak{K}_{n}$ is residually a $\mathcal{C}$-group, and $\mathcal{C}_{\cap}^{*}\left(K_{n}, H_{n+1}, K_{n+1}, \varphi\right)$ is a filtration by Proposition 5.2. Because $H_{n+1}=K_{n}=K_{n+1}$, this family is actually an $\left(H_{n+1}, K_{n+1}\right)$-filtration. By Proposition 3.2, it is closed under taking finite intersections and therefore is a strong $\left(H_{n+1}, K_{n+1}\right)$-filtration. It follows from Proposition 3.5 that $\mathcal{C}_{\cap}^{*}\left(K_{n}, H_{n+1}, K_{n+1}, \varphi\right)=\mathcal{C}_{n}^{*}\left(K_{n}, H_{n+1}, K_{n+1}, \varphi\right)$. Hence, $\mathcal{C}_{n}^{*}\left(K_{n}, H_{n+1}, K_{n+1}, \varphi\right)$ is also a strong $\left(H_{n+1}, K_{n+1}\right)$-filtration.

Since $G$ is residually a $\mathcal{C}$-group and $\mathcal{C}$ is closed under taking subgroups, then $K_{i}(i \geqslant 0)$ are also residually $\mathcal{C}$-groups. For any $i \in\{0,1, \ldots, n-1\}, P_{i+1}$ is central in $K_{i}$. Hence, by Proposition 6.4, the $\pi(\mathcal{C})^{\prime}$-isolator $X_{i}=\mathcal{I}_{\pi(\mathcal{C})^{\prime}}\left(K_{i}, P_{i+1}\right)$ lies in the center of $K_{i}$ and coincides with the set $\mathcal{R}_{\pi(\mathcal{C})^{\prime}}\left(K_{i}, P_{i+1}\right)$. Therefore, $X_{i} / P_{i+1}$ is a periodic $\pi(\mathcal{C})^{\prime}$-group.

By the hypothesis of the theorem, $X_{i}$ is $\mathcal{C}$-separable in $K_{i}$ for any $i \in\{0,1, \ldots, n-1\}$, and therefore $K_{i} / X_{i}$ is residually a $\mathcal{C}$-group by Proposition 6.5. Again by the hypothesis, $K_{i}$ is $\mathcal{C}$-regular with respect to $P_{i+1}$. By Proposition $6.2, H_{i+1}$ and $K_{i+1}$ are $\pi(\mathcal{C})^{\prime}$-isolated in $G$ and therefore in $K_{i}$. Hence, we can successively apply Proposition 4.4 to the HNNtuples $\left(K_{i}, H_{i+1}, K_{i+1}, \varphi\right), i=n-1, \ldots, 1,0$. As a result, we see that $\mathcal{C}_{0}^{*}(G, H, K, \varphi)$ is a strong $(H, K)$-filtration. By Proposition 3.4, $\mathcal{C}_{0}^{*}(G, H, K, \varphi) \subseteq \mathcal{C}_{\cap}^{*}(G, H, K, \varphi)$. Therefore, $\mathcal{C}_{\cap}^{*}(G, H, K, \varphi)$ is an $(H, K)$-filtration, and $\mathfrak{G}$ is residually a $\mathcal{C}$-group by Proposition 5.2 .

Proof of Theorem 4. Since $G$ is residually a $\mathcal{C}$-group and $\mathcal{C}$ is closed under taking subgroups, then $K_{n}$ is also residually a $\mathcal{C}$-group and, by Proposition $5.5, \mathcal{C}^{*}\left(K_{n}, H_{n+1}, K_{n+1}, \varphi\right)$ is an $\left(H_{n+1}, K_{n+1}\right)$-filtration. By Proposition 3.2, this family is closed under taking finite intersections and therefore turns out to be a strong $\left(H_{n+1}, K_{n+1}\right)$-filtration. By the hypothesis of the theorem, $P_{i+1}$ is $\mathcal{C}$-separable in $K_{i}$ for each $i \in\{0,1, \ldots, n-1\}$. Hence, $K_{i} / P_{i+1}$ is residually a $\mathcal{C}$-group by Proposition 6.5 . If we take the set of all primes as $\pi$ and successively apply Proposition 4.4 to the HNN-tuples $\left(K_{i}, H_{i+1}, K_{i+1}, \varphi\right)$ and the subgroups $X_{i+1}=P_{i+1}(i=n-1, \ldots, 1,0)$, then we see that $\mathcal{C}^{*}(G, H, K, \varphi)$ is a strong $(H, K)$-filtration. Since $\mathcal{C}^{*}(G, H, K, \varphi)=\mathcal{C}_{\cap}^{*}(G, H, K, \varphi)$ by Proposition 3.4, then $\mathcal{C}_{\cap}^{*}(G, H, K, \varphi)$ is an $(H, K)$-filtration. Thus, $\mathfrak{G}$ is residually a $\mathcal{C}$-group by Proposition 5.2 .

Proof of Theorem 1. Statement I follows from Theorem 4. Let us prove Statement II.

We take a number $i \geqslant 0$ and show that $K_{i} / P_{i+1} \in \mathcal{C}$.

If $Q \leqslant H \cap K$ and $Q \varphi=Q$, then $Q \leqslant H_{i+1} \cap K_{i+1}$ by Proposition 6.2 and $Q \leqslant P_{i+1}$. Hence, $G / P_{i+1} \cong(G / Q) /\left(P_{i+1} / Q\right) \in \mathcal{C}$ and $K_{i} / P_{i+1} \in \mathcal{C}$ because $\mathcal{C}$ is closed under taking subgroups and quotient groups. If $H \cap Q=1=K \cap Q$, then $K_{i} \cap Q=1$ 
and $K_{i} / P_{i+1} \cong K_{i} / P_{i+1}\left(K_{i} \cap Q\right) \cong K_{i} Q / P_{i+1} Q$. Since $Q \leqslant P_{i+1} Q$, then, as above, $K_{i} Q / P_{i+1} Q \in \mathcal{C}$.

Thus, $K_{i} / P_{i+1} \in \mathcal{C}$. It follows that $P_{i+1}$ is $\mathcal{C}$-separable in $K_{i}$ and, by Proposition 6.3 , is $\pi(\mathcal{C})^{\prime}$-isolated in this group. Therefore, $P_{i+1}=\mathcal{I}_{\pi(\mathcal{C})^{\prime}}\left(K_{i}, P_{i+1}\right)$. We note also that if $N \in \mathcal{C}^{*}\left(P_{i+1}\right)$, then $K_{i} / N$ is an extension of the $\mathcal{C}$-group $P_{i+1} / N$ by the $\mathcal{C}$-group $K_{i} / P_{i+1}$ and $K_{i} / N \in \mathcal{C}$ because $\mathcal{C}$ is closed under taking extensions. Hence, $\mathcal{C}^{*}\left(P_{i+1}\right) \subseteq \mathcal{C}^{*}\left(K_{i}\right)$ and therefore $K_{i}$ is $\mathcal{C}$-regular with respect to $P_{i+1}$.

Thus, the statement to be proved follows from Theorem 3. We only need to note that $H$ and $K$ are $\mathcal{C}$-separable in $G$ by Proposition 5.4 and are $\pi(\mathcal{C})^{\prime}$-isolated in this group by Proposition 6.3.

Proof of Corollary 1. Because $H$ is finite, the equalities $H_{n}=H_{n+1}=H_{n} \cap K_{n}$ hold for some $n \geqslant 1$. Therefore, $H_{n} \leqslant K_{n}$. But the subgroups $H_{n}$ and $K_{n}$ are finite and isomorphic, hence, $H_{n}=K_{n}$. Since $G$ is residually a $\mathcal{C}$-group, then, by Proposition 3.1, there exists a subgroup $Q \in \mathcal{C}^{*}(G)$ such that $H \cap Q=1=K \cap Q$. Therefore, Statement I follows from Theorem 1. Let us prove Statement II.

Since $H \cap Q=1$, then $H_{n}$ is embedded into the $\mathcal{C}$-group $G / Q$ and belongs to $\mathcal{C}$ because this class is closed under taking subgroups. The restriction of $\varphi$ to the finite subgroup $H_{n}$ has a finite order $q$. Hence, by Proposition 5.8, the subgroup $E=\operatorname{sgp}\left\{H_{n}, t\right\}$ of $\mathfrak{G}$ is residually a $\mathcal{C}$-group if and only if $q$ is a $\pi(\mathcal{C})$-number. If $H=G=K$, then $\mathfrak{G}=E$, and the required statement is proved. Otherwise, the inequalities $G \neq H$ and $G \neq K$ hold simultaneously because $H$ and $K$ are finite and isomorphic. Therefore, the statement of the corollary follows from Theorem 1 .

Proof of Corollary 2. I. The conditions of the statement are necessary for $\mathfrak{G}$ to be residually a $\mathcal{C}$-group by Proposition 5.6 and because $\mathcal{C}$ is closed under taking subgroups. Their sufficiency follows from Theorem 1.

II. Necessity. By Propositions 5.6 and 6.2, there exists a subgroup $Q \in \mathcal{C}^{*}(G)$ such that $Q \varphi=Q$ and $Q \leqslant H_{i} \cap K_{i}$ for all $i \geqslant 1$. Since $\mathcal{C}$ consists of finite groups, $Q$ has a finite index in $G$ and therefore $H_{n}=H_{n+1}$ for some $n$. Because $\mathcal{C}$ is closed under taking subgroups, $G$ is residually a $\mathcal{C}$-group. Therefore, Conditions 2 and 3 follow from Theorem 1 . Since $\mathcal{C}$ is closed under taking quotient groups, Condition 1 follows from the relations

$$
G / H \cong(G / Q) /(H / Q), \quad G / K \cong(G / Q) /(K / Q) .
$$

Sufficiency. By Proposition 6.2, $H_{i} \in \mathcal{C}^{*}(G)$ and $K_{i} \in \mathcal{C}^{*}(G)$ for any $i \geqslant 1$. Hence, $H_{n} \in \mathcal{C}^{*}(G), H_{n} \leqslant H \cap K$, and $H_{n} \varphi=H_{n}$. Since $E$ is residually a $\mathcal{C}$-group and $\mathcal{C}$ is closed under taking subgroups, then $H_{n}$ is also residually a $\mathcal{C}$-group. Therefore, $G$ is an extension of the residually $\mathcal{C}$-group $H_{n}$ by the $\mathcal{C}$-group $G / H_{n}$. Such an extension is residually a $\mathcal{C}$-group by Proposition 5.1. Thus, it follows from Theorem 1 that $\mathfrak{G}$ is residually a $\mathcal{C}$-group.

Proposition 6.6. [25, Proposition 18] If $\mathcal{C}$ is a root class of groups consisting only of periodic groups and closed under taking quotient groups, then any $\pi(\mathcal{C})$-bounded solvable $\mathcal{C}$-group is finite.

Proposition 6.7. [16, Propositions 1, 2, 3] If $\pi$ is a non-empty set of primes, then the following statements hold.

1. Any $\pi$-bounded abelian group is of finite rank.

2. The classes of $\pi$-bounded abelian, $\pi$-bounded nilpotent, and $\pi$-bounded solvable groups are closed under taking subgroups and quotient groups.

3. If a $\pi$-bounded solvable group is abelian, then it belongs to the class of $\pi$-bounded abelian groups. 
Proof of Theorem 2. Let us show that, for any $i \in\{0,1, \ldots, n-1\}, K_{i}$ is $\mathcal{C}$-regular with respect to $P_{i+1}$. Then the required statement will follow from Theorem 3 .

Suppose that $i \in\{0,1, \ldots, n-1\}$ and $M \in \mathcal{C}^{*}\left(P_{i+1}\right)$. We need to find a subgroup $N \in \mathcal{C}^{*}\left(K_{i}\right)$ such that $N \cap P_{i+1}=M$.

Let us denote the $\pi(\mathcal{C})^{\prime}$-isolator $\mathcal{I}_{\pi(\mathcal{C})^{\prime}}\left(K_{i}, M\right)$ by $\mathfrak{I}$ for brevity. Since $G$ is residually a $\mathcal{C}$-group and $\mathcal{C}$ is closed under taking subgroups, then $K_{i}$ is also residually a $\mathcal{C}$-group. Therefore, by Proposition 6.4, $\mathfrak{I}$ lies in the center of $K_{i}$ and coincides with the set $\mathcal{R}_{\pi(\mathcal{C})^{\prime}}\left(K_{i}, M\right)$. It follows from the equality $\mathfrak{I}=\mathcal{R}_{\pi(\mathcal{C})^{\prime}}\left(K_{i}, M\right)$ that, if $x \in \mathfrak{I} \cap P_{i+1}$, then $x^{q} \in M$ for some $\pi(\mathcal{C})^{\prime}$-number $q$. But the quotient group $P_{i+1} / M$ belongs to $\mathcal{C}$ and therefore has no $\pi(\mathcal{C})^{\prime}$-torsion. Hence, $x M=1$ and $x \in M$. Thus, $\mathfrak{I} \cap P_{i+1} \leqslant M$ and, because the opposite inclusion is obvious, $\mathfrak{I} \cap P_{i+1}=M$.

Since $H$ and $K$ are $\pi(\mathcal{C})$-bounded, then it follows from Propositions 6.6 and 6.7 that the $\mathcal{C}$-group $P_{i+1} / M$ is $\pi(\mathcal{C})$-bounded and therefore finite. Hence, the subgroup

$$
P_{i+1} \mathfrak{I} / \mathfrak{I} \cong P_{i+1} /\left(P_{i+1} \cap \mathfrak{I}\right)=P_{i+1} / M
$$

is also finite. By the hypothesis of the theorem, $\mathfrak{I}$ is $\mathcal{C}$-separable in $K_{i}$. Therefore, by Proposition $6.5, K_{i} / \mathfrak{I}$ is residually a $\mathcal{C}$-group and, by Proposition 3.1 , there exists a subgroup $N / \mathfrak{I} \in \mathcal{C}^{*}\left(K_{i} / \mathfrak{I}\right)$ such that $N / \mathfrak{I} \cap P_{i+1} \mathfrak{I} / \mathfrak{I}=1$. Then $N \in \mathcal{C}^{*}\left(K_{i}\right)$ and, as it is easy to see, $N \cap P_{i+1}=M$. Thus, $N$ is the required subgroup.

Proposition 6.8. Suppose that $\mathcal{C}$ is a root class of groups consisting only of periodic groups and closed under taking quotient groups, $H \neq G \neq K$, and there exists $m \geqslant 0$ such that at least one of the following conditions holds:

( $\alpha) H_{m+1}$ and $K_{m+1}$ are finitely generated;

( $\beta) H_{m+1}$ and $K_{m+1}$ are $\pi(\mathcal{C})$-bounded and $\pi^{\prime}$-isolated in $K_{m}$ for some finite subset $\pi$ of $\pi(\mathcal{C})$.

If $\mathfrak{G}$ is residually a $\mathcal{C}$-group, then $H_{n}=H_{n+1}$ for some $n$.

Proof. Because $\mathcal{C}$ contains non-trivial groups, $\pi(\mathcal{C})$ is non-empty. Hence, the rank rk $H_{m+1}$ of $H_{m+1}$ is finite by Proposition 6.7, and since rk $H_{i+1} \leqslant \operatorname{rk} H_{i}$ for any $i \geqslant 1$, then $\operatorname{rk} H_{l}=\operatorname{rk} H_{l+1}$ for some $l \geqslant m+1$. It follows from the relations $H_{l} \cong K_{l}$ and $H_{l+1} \cong K_{l+1}$ that

$$
\operatorname{rk} K_{l}=\operatorname{rk} H_{l}=\operatorname{rk} H_{l+1}=\operatorname{rk} K_{l+1}
$$

and therefore the quotient groups $K_{l} / H_{l+1}$ and $K_{l} / K_{l+1}$ are periodic. If $H_{m+1}$ and $K_{m+1}$ are finitely generated, then $K_{l} / H_{l+1}$ and $K_{l} / K_{l+1}$ are finite. Let us show that this is also true if $(\beta)$ holds.

Since $H_{m+1}$ and $K_{m+1}$ are $\pi^{\prime}$-isolated in $K_{m}$, then, by Proposition 6.2 applied to the HNN-tuple $\left(K_{m}, H_{m+1}, K_{m+1}, \varphi\right), H_{l+1}$ and $K_{l+1}$ are also $\pi^{\prime}$-isolated in this group. Hence, $K_{l} / H_{l+1}$ and $K_{l} / K_{l+1}$ are periodic $\pi$-groups, and each of them has a finite number of primary components because $\pi$ is finite. By Proposition 6.7, these groups are $\pi(\mathcal{C})$-bounded, and since $\pi \subseteq \pi(\mathcal{C})$, all their primary components are finite. Thus, $K_{l} / H_{l+1}$ and $K_{l} / K_{l+1}$ are also finite.

By Proposition 6.1, the HNN-extensions

$$
\mathfrak{G}_{l}=\operatorname{HNN}\left(G, H_{l+1}, K_{l+1}, \varphi\right), \quad \mathfrak{K}_{l}=\operatorname{HNN}\left(K_{l}, H_{l+1}, K_{l+1}, \varphi\right)
$$

are residually $\mathcal{C}$-groups. If $H_{l+1}=K_{l}$ or $K_{l+1}=K_{l}$, then $H_{l+1} \geqslant K_{l+1}$ or $H_{l+1} \leqslant K_{l+1}$ and it follows from Proposition 5.3 applied to $\mathfrak{G}_{l}$ that $H_{l+1}=K_{l+1}$. Hence, we can put $n=l+1$. Let $H_{l+1} \neq K_{l} \neq K_{l+1}$. Then, by Proposition 5.6 applied to $\mathfrak{K}_{l}$, there exists a subgroup $Q \in \mathcal{C}^{*}\left(K_{l}\right)$ such that $Q \leqslant H_{l+1} \cap K_{l+1}$ and $Q \varphi=Q$. The $\mathcal{C}$-group $K_{l} / Q$ is finite: this is obvious if $(\alpha)$ holds, and follows from Propositions 6.6, 6.7 if $(\beta)$ takes place. By Proposition 6.2, $Q \leqslant H_{i}$ for any $i \geqslant l+1$. Thus, there exists $n \geqslant l+1$ such that $H_{n}=H_{n+1}$. 
Proposition 6.9. [18, Proposition 10] If $\mathcal{C}$ is a root class of groups consisting only of periodic groups, then any finite solvable $\pi(\mathcal{C})$-group belongs to $\mathcal{C}$.

Proposition 6.10. If $\mathcal{C}$ is a root class of groups consisting only of periodic groups and $\pi(\mathcal{C})$ contains all prime numbers, then all the subgroups of an arbitrary $\pi(\mathcal{C})$-bounded solvable group are $\mathcal{C}$-separable.

Proof. Let $X$ be a $\pi(\mathcal{C})$-bounded solvable group, and let $Y$ be a subgroup of $X$. By Theorem 6 from [10], $Y$ is $\mathcal{F}$-separable in $X$, where $\mathcal{F}$ is the class of all finite groups. Since any homomorphic image of $X$ is a solvable group, then $Y$ turns out to be $\mathcal{F} \mathcal{S}$-separable in $X$, where $\mathcal{F} \mathcal{S}$ is the class of all finite solvable groups. By Proposition $6.9, \mathcal{F} \mathcal{S} \subseteq \mathcal{C}$. Hence, $Y$ is $\mathcal{C}$-separable.

Proposition 6.11. [22, Proposition 8] If $\mathcal{C}$ is a root class of groups consisting only of periodic groups and $X$ is a $\pi(\mathcal{C})$-bounded nilpotent group, then every $\pi(\mathcal{C})^{\prime}$-isolated subgroup of $X$ is $\mathcal{C}$-separable in this group.

Proof of Corollaries 3 and 4. Let us verify that the conditions of Theorem 2 hold.

By Proposition 6.7, $H_{i}$ and $K_{i}(i \geqslant 1)$ are $\pi(\mathcal{C})$-bounded abelian groups. Therefore, it follows from Propositions 6.10 and 6.11 that all the $\pi(\mathcal{C})^{\prime}$-isolated subgroups of $K_{i}(i \geqslant 0)$ are $\mathcal{C}$-separable in this group. In particular, if $N \in \mathcal{C}^{*}\left(P_{i+1}\right)$, then the subgroup $\mathcal{I}_{\pi(\mathcal{C})^{\prime}}\left(K_{i}, N\right)$ is $\mathcal{C}$-separable in $K_{i}$.

The fact that $G$ is residually a $\mathcal{C}$-group follows from

- Proposition 6.10 if $\pi(\mathcal{C})$ contains all primes and $G$ is a $\pi(\mathcal{C})$-bounded solvable group;

- Proposition 6.11 and the absence of $\pi(\mathcal{C})^{\prime}$-torsion in $G$ if the last group is $\pi(\mathcal{C})$-bounded nilpotent;

- the assumption that $\mathcal{C}$ is closed under taking subgroups if $\mathfrak{G}$ is residually a $\mathcal{C}$-group.

Finally, $H_{n}=H_{n+1}$ for some $n \geqslant 1$ : it is obvious if $H_{n}=K_{n}$, and is guaranteed by Proposition 6.8 if $\mathfrak{G}$ is residually a $\mathcal{C}$-group. Thus, the necessity of the statements of both corollaries follows from Theorem 2. We only need to note that if $\mathfrak{G}$ is residually a $\mathcal{C}$-group, then $G$ is also residually a $\mathcal{C}$-group and, by Proposition 6.3 , has no $\pi(\mathcal{C})^{\prime}$-torsion.

The group $E$ is $\pi(\mathcal{C})$-bounded solvable as an extension of the $\pi(\mathcal{C})$-bounded abelian group $H_{n}$ by the infinite cyclic group $\langle t\rangle$, which is also $\pi(\mathcal{C})$-bounded abelian. If $\pi(\mathcal{C})$ contains all primes, then $H$ and $K$ are $\pi(\mathcal{C})^{\prime}$-isolated in $G$ and, by Proposition 6.10, $E$ is residually a $\mathcal{C}$-group. Therefore, the sufficiency of the statements of the corollaries also follows from Theorem 2.

\section{REFERENCES}

[1] Allenby R. B. J. T. The potency of cyclically pinched one-relator groups, Arch. Math. 36 (1981) 204-210.

[2] Andreadakis S., Raptis E., Varsos D. A characterization of residually finite HNN-extensions of finitely generated Abelian groups, Arch. Math. 50 (6) (1988) 495-501.

[3] Azarov D. N., Tieudjo D. On the root-class residuality of a free product of groups with an amalgamated subgroup, Nauch. Tr. Ivanov. Gos. Univ. Math. 5 (2002) 6-10 (in Russian). See also: Azarov D. N., Tieudjo D. On root-class residuality of generalized free products, arXiv:math.GR/0408277.

[4] Baumslag B., Tretkoff M. Residually finite HNN-extensions, Comm. Algebra 6 (2) (1978) 179-194.

[5] Cohen D. E. Subgroups of HNN groups, J. Austral. Math. Soc. 17 (1974) 394-405.

[6] Gol'tsov D. V. Approximability of HNN-extensions with central associated subgroups by a root class of groups, Math. Notes 97 (5) (2015) 679-683.

[7] Gruenberg K. W. Residual properties of infinite soluble groups, Proc. London Math. Soc. s3-7 (1) (1957) 29-62.

[8] Kim G., Tang C. Y. Cyclic subgroup separability of HNN-extensions with cyclic associated subgroups, Can. Math. Bull. 42 (3) (1999) 335-343. 
[9] Lyndon R. C., Schupp P. E. Combinatorial group theory (Springer-Verlag, New York, 1977).

[10] Mal'cev A. I. On homomorphisms onto finite groups, Ivanov. Gos. Ped. Inst. Ucen. Zap. 18 (1958) 49-60 (in Russian). See also: Mal'cev A. I. On homomorphisms onto finite groups, Transl. Am. Math. Soc. 2 (119) (1983) 67-79.

[11] Moldavanskii D. I. The residual finiteness of some HNN-extensions of groups, Bull. Ivanovo State Univ. (3) (2002) 123-133 (in Russian).

[12] Moldavanskii D. I. The residual $p$-finiteness of some HNN-extensions of groups, Bull. Ivanovo State Univ. (3) (2003) 102-116 (in Russian).

[13] Raptis E., Varsos D. Some residual properties of certain HNN extensions, Bull. Greek Math. Soc. 28 (1987) 81-87.

[14] Raptis E., Varsos D. Residual properties of HNN-extensions with base group an Abelian group, J. Pure Appl. Algebra 59 (3) (1989) 285-290.

[15] Raptis E., Varsos D. The residual nilpotence of HNN-extensions with base group a finite or a f. g. abelian group, J. Pure Appl. Algebra 76 (2) (1991) 167-178.

[16] Sokolov E. V. Separability of subgroups of nilpotent groups in the class of finite $\pi$-groups, Sib. Math. J. 55 (6) (2014) 1126-1132.

[17] Sokolov E. V. A characterization of root classes of groups, Comm. Algebra 43 (2) (2015) 856-860.

[18] Sokolov E. V. Separability of the subgroups of residually nilpotent groups in the class of finite $\pi$-groups, Sib. Math. J. 58 (1) (2017) 169-175.

[19] Sokolov E. V. Certain residual properties of generalized Baumslag-Solitar groups, J. Algebra 582 (2021) $1-25$.

[20] Sokolov E. V. On the root-class residuality of the fundamental groups of graph of groups, To appear in Sib. Math. J.

[21] Sokolov E. V. On the root-class residuality of the fundamental groups of certain graph of groups with central edge subgroups, Submitted to Sib. Math. J.

[22] Sokolov E. V., Tumanova E. A. Sufficient conditions for the root-class residuality of certain generalized free products, Sib. Math. J. 57 (1) (2016) 135-144.

[23] Sokolov E. V., Tumanova E. A. Root class residuality of HNN-extensions with central cyclic associated subgroups, Math. Notes 102 (4) (2017) 556-568.

[24] Sokolov E. V., Tumanova E. A. Generalized direct products of groups and their application to the study of residuality of free constructions of groups, Algebra Logic 58 (6) (2020) 480-493.

[25] Sokolov E. V., Tumanova E. A. On the root-class residuality of certain free products of groups with normal amalgamated subgroups, Russ. Math. 64 (2020) 43-56.

[26] Sokolov E. V., Tumanova E. A. The root-class residuality of tree products with central amalgamated subgroups, Sib. Math. J. 61 (3) (2020) 545-551.

[27] Sokolov E. V., Tumanova E. A. To the question of the root-class residuality of free constructions of groups, Lobachevskii J. Math. 41 (2020) 260-272.

[28] Tieudjo D. On root-class residuality of some free constructions, JP J. Algebra, Number Theory Appl. 18 (2) (2010) 125-143.

[29] Tumanova E. A. On the root-class residuality of HNN-extensions of groups, Model. Anal. Inform. Syst. 21 (4) (2014) 148-180 (in Russian).

[30] Tumanova E. A. The root class residuality of Baumslag-Solitar groups, Sib. Math. J. 58 (3) (2017) $546-552$.

[31] Tumanova E. A. The root class residuality of the tree product of groups with amalgamated retracts, Sib. Math. J. 60 (4) (2019) 699-708.

[32] Tumanova E. A. On the root-class residuality of certain HNN-extensions of groups, Russ. Math. 64 (2020) 38-45.

[33] Wong P. C., Gan H.W. Cyclic subgroup separability of certain HNN extensions, Bull. Malaysian Math. Soc. (Second Ser.) 22 (2) (1999) 169-177.

[34] Wong P. C., Wong K. B. The residual finiteness of certain HNN extensions, Bull. Korean Math. Soc. $42(3)(2005) 555-561$.

IVAnovo State University, Russia

Email address: ev-sokolov@yandex.ru 